\title{
Squeezed between shells? The origin of the Lupus I molecular cloud
}

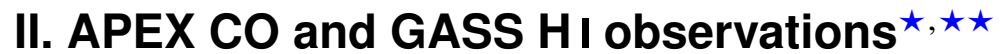

\author{
B. Gaczkowski ${ }^{1}$, V. Roccatagliata ${ }^{2,1}$, S. Flaischlen ${ }^{1}$, D. Kröll ${ }^{1,3}$, M. G. H. Krause ${ }^{5,1,3,4,6}$, A. Burkert ${ }^{1,3}$, R. Diehl ${ }^{3,4}$, \\ K. Fierlinger ${ }^{1,4}$, J. Ngoumou ${ }^{1}$, and T. Preibisch ${ }^{1}$
}

1 Universitäts-Sternwarte München, Ludwig-Maximilians-Universität, Scheinerstr. 1, 81679 München, Germany
e-mail: bengac@usm.uni-muenchen.de, roccatagliata@arcetri.astro.it
2 INAF-Osservatorio Astrofisico di Arcetri, L.go E. Fermi 5, 50125 Firenze, Italy
3 Max-Planck-Institut für extraterrestrische Physik, Postfach 1312, 85741 Garching, Germany
4 Excellence Cluster Universe, Boltzmannstrasse 2, 85748 Garching, Germany
5 School of Mathematics \& Physics, Private Bag 37, University of Tasmania, Hobart 7001, Australia
6 Centre for Astrophysics Research, School of Physics, Astronomy and Mathematics, University of Hertfordshire, College Lane,
Hatfield, Hertfordshire AL10 9AB, UK

Received 14 March 2016 / Accepted 13 October 2017

\begin{abstract}
Context. Lupus I cloud is found between the Upper Scorpius (USco) and Upper Centaurus-Lupus (UCL) subgroups of the ScorpiusCentaurus OB association, where the expanding USco H I shell appears to interact with a bubble currently driven by the winds of the remaining B stars of UCL.

Aims. We investigate whether the Lupus I molecular could have formed in a colliding flow, and in particular, how the kinematics of the cloud might have been influenced by the larger scale gas dynamics.

Methods. We performed APEX ${ }^{13} \mathrm{CO}(2-1)$ and $\mathrm{C}^{18} \mathrm{O}(2-1)$ line observations of three distinct parts of Lupus I that provide kinematic information on the cloud at high angular and spectral resolution. We compare those results to the atomic hydrogen data from the GASS H I survey and our dust emission results presented in the previous paper. Based on the velocity information, we present a geometric model for the interaction zone between the USco shell and the UCL wind bubble.

Results. We present evidence that the molecular gas of Lupus I is tightly linked to the atomic material of the USco shell. The $\mathrm{CO}$ emission in Lupus I is found mainly at velocities between $v_{\mathrm{LSR}}=3-6 \mathrm{~km} \mathrm{~s}^{-1}$, which is in the same range as the H I velocities. Thus, the molecular cloud is co-moving with the expanding USco atomic H I shell. The gas in the cloud shows a complex kinematic structure with several line-of-sight components that overlay each other. The nonthermal velocity dispersion is in the transonic regime in all parts of the cloud and could be injected by external compression. Our observations and the derived geometric model agree with a scenario in which Lupus I is located in the interaction zone between the USco shell and the UCL wind bubble.

Conclusions. The kinematics observations are consistent with a scenario in which the Lupus I cloud formed via shell instabilities. The particular location of Lupus I between USco and UCL suggests that counterpressure from the UCL wind bubble and pre-existing density enhancements, perhaps left over from the gas stream that formed the stellar subgroups, may have played a role in its formation.
\end{abstract}

Key words. ISM: bubbles - ISM: clouds - ISM: kinematics and dynamics - ISM: structure - ISM: molecules - radio lines: ISM

\section{Introduction}

Molecular cloud formation is attributed to collisions of largescale flows in the interstellar medium (ISM) that can be driven by stellar feedback processes such as UV radiation, winds, and supernovae (see review by Dobbs et al. 2014). At the interface of the colliding flows, compression, cooling, and fragmentation of the diffuse atomic medium produces cold sheets and filaments that later may become molecular and self-gravitating. Realizations of this general concept include expanding (super)

^ This publication is based on data acquired with the Atacama Pathfinder Experiment (APEX), which is a collaboration between the Max-Planck-Institut fur Radioastronomie, the European Southern Observatory, and the Onsala Space Observatory.

$\star \star$ The $13 \mathrm{CO}(2-1)$ and $\mathrm{C} 18 \mathrm{O}(2-1)$ spectral cubes are only available at the CDS via anonymous ftp to

cdsarc.u-strasbg. fr (130.79.128.5) or via

http://cdsarc.u-strasbg.fr/viz-bin/qcat?J/A+A/608/A102 shells, where an expanding hot bubble collides with the ambient medium (e.g., Krause et al. 2013) and direct collisions of stellar winds (e.g., Calderón et al. 2016).

Such a process might take place between the Upper Scorpius (USco) H I shell and the Upper Centaurus-Lupus (UCL) wind bubble located in the Scorpius-Centaurus OB association (Blaauw 1964; de Zeeuw et al. 1999; Preibisch et al. 2002; Preibisch \& Mamajek 2008, Sco-Cen). This is the closest site of recent massive star formation to us at a distance of 110-140 pc only. These shells presumably were created by the feedback of the numerous massive stars in Sco-Cen. In between those two expanding structures there is the Lupus I cloud, which represents a good candidate for which we can study how such a collision process forms and influences new dense clouds in the ISM.

The distance of the Lupus cloud complex is about $140 \mathrm{pc}$, as discussed in detail by Comerón (2008). Additional details about the region and Lupus I cloud can be found in Gaczkowski et al. (2015, Paper I hereafter). 
Molecular line observations of Lupus I with different CO isotopes and other high-density tracers have been performed by several groups over the past two decades. Most of these observations have spatial resolutions of several arcminutes, i.e., $\sim 0.1 \mathrm{pc}$ at the distance of Lupus I of $\sim 140 \mathrm{pc}$. Tachihara et al. (1996) observed the entire Lupus I cloud in the ${ }^{13} \mathrm{CO}(1-0)$. These authors estimated the cloud mass to be $\sim 1200 M_{\odot}$ and discovered the molecular outflow of IRAS source 15398-3359. Hara et al. (1999) have studied 12 cores in Lupus I in the $\mathrm{C}^{18} \mathrm{O}(1-0)$ line. The spatial resolution of the NANTEN telescope is $2.7^{\prime}$, while their grid spacing was $2^{\prime}$. These authors derived column densities, temperatures, and sizes for the cores and estimated their total mass to be $152 M_{\odot}$. This represents $46 \%$ of their total $\mathrm{C}^{18} \mathrm{O}(1-0)$ emitting gas mass. Vilas-Boas et al. (2000) found 15 condensations in $\mathrm{C}^{18} \mathrm{O}(1-0)$, and 14 in ${ }^{13} \mathrm{CO}(1-0)$, highlighting the recent star formation activity in Lupus I. A large-scale ${ }^{12} \mathrm{CO}(1-0)$ survey of Lupus I was performed by Tachihara et al. (2001). They found a velocity gradient along the long axis of Lupus I from $4 \mathrm{~km} \mathrm{~s}^{-1}$ in the south to $6 \mathrm{~km} \mathrm{~s}^{-1}$ in the north. Tothill et al. (2009) analyzed Lupus I in both $\mathrm{CO}(4-3)$ and ${ }^{13} \mathrm{CO}(2-1)$. They found several possible signs of interaction between Lupus I and the USco H I shell. Benedettini et al. (2012) found eight dense cores in Lupus I using high-density molecular tracers at 3 and $12 \mathrm{~mm}$. They reported the presence of velocity gradients in the cloud and several velocity components along the line of sight. Finally, Benedettini et al. (2015) observed the southern part of the main filament in $\operatorname{CS}(2-1)$ and they found several subfilaments in velocity space that correspond to subfilaments they had identified in their Herschel column density maps.

In our dust analysis presented in Paper I we found a doublepeaked PDF, which suggests a scenario of external compression of Lupus I from USco and/or UCL. In this work, we follow up on this suggestion and present ${ }^{13} \mathrm{CO}(2-1)$ and $\mathrm{C}^{18} \mathrm{O}(2-1)$ observations of three distinct regions within the Lupus I cloud with a high angular and spectral resolution of $\approx 30^{\prime \prime}$ and $\approx 0.1 \mathrm{~km} \mathrm{~s}^{-1}$, respectively. We present an analysis of the observational data and a comparison to the available H I data. From those we create a geometrical model for the interaction zone of shell and bubble. We show that the collision scenario is a plausible explanation of the observational data and the creation of Lupus I with other factors likely having played a role as well.

\section{Observations and data reduction}

\subsection{APEX CO observations}

The CO line observations of Lupus I were performed with the APEX telescope (Güsten et al. 2006) using the APEX1 receiver of the Swedish Heterodyne Facility Instrument (SHeFI; Vassilev et al. 2008). The observations were carried out on 18 and 20 August 2014 (PI: B. Gaczkowski; Program ID: 093.F-9311(A)). Lupus I was observed simultaneously in the ${ }^{13} \mathrm{CO}(2-1)$ (rest frequency $v_{0}^{13}=220.398677 \mathrm{GHz}$ ) and $\mathrm{C}^{18} \mathrm{O}(2-1)\left(v_{0}^{18}=219.560357 \mathrm{GHz}\right)$ line. Three scans across the cloud were performed, as shown in Fig. 1. These were chosen based on our previous dust analysis to obtain representative samples of the conditions along the whole filament. The observing mode was "on-the-fly" and the map parameters for each cut are listed in Table 1. The scanning direction was along the long side of a cut. The velocity resolution of the spectra is $\Delta v=0.1 \mathrm{~km} \mathrm{~s}^{-1}$ and the rms noise ranges between $\approx 0.05-0.1 \mathrm{~K}$ for both lines. The perceptible water vapor (PWV) during the observations was between 1.5 and $2 \mathrm{~mm}$. Each cut contains about 620 spectra.

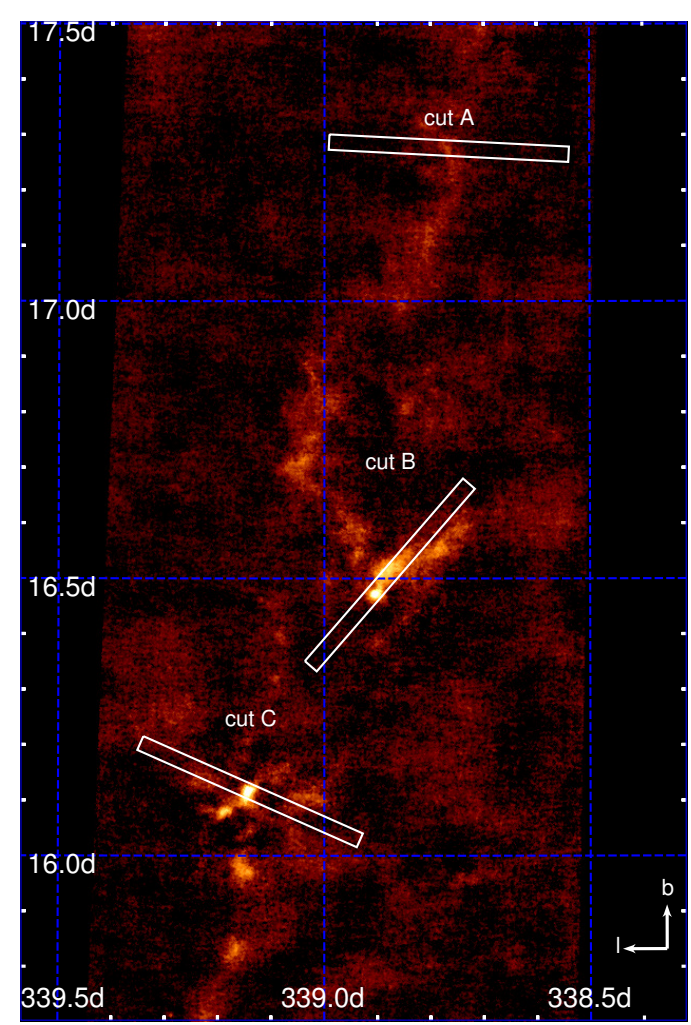

Fig. 1. Setup of the APEX-1 ${ }^{13} \mathrm{CO}(2-1)$ and $\mathrm{C}^{18} \mathrm{O}(2-1)$ observations of Lupus I. Along each of the three cuts A, B, and C an on-the-fly map was obtained simultaneously in both lines. The size of each map is indicated with a white box. The background image is the LABOCA continuum map (see Paper I). The Galactic coordinate system is indicated.

The data reduction was carried out with the CLASS package of the IRAM GILDAS software ${ }^{1}$. The antenna temperature $T_{\mathrm{A}}$ was converted into a main-beam temperature $T_{\mathrm{mb}}=T_{\mathrm{A}} / \eta$, adopting a main-beam efficiency of $\eta=0.75$ for APEX-1 ${ }^{2}$. All spectra were baseline subtracted. Finally, for each line and cut, a map (or lmv cube) was produced with the XY_MAP task, which convolves the data with a Gaussian of one-third of the beam. The final angular resolution is $30.1^{\prime \prime}$ and the pixel size is $14.3^{\prime \prime}$.

\subsection{GASS Hı data}

The atomic hydrogen data of the entire Sco-Cen region used in our study are part of the second data release of the GASS H I survey (Kalberla et al. 2010). This has a spatial resolution of $16.2^{\prime}$, which corresponds to a length scale of $0.7 \mathrm{pc}$ at the distance of Lupus I. The velocity resolution is $1 \mathrm{~km} \mathrm{~s}^{-1}$ and the rms noise is $55 \mathrm{mK}$.

In order to characterize the HI shell around Upper Scorpius (the Upper Scorpius loop) and relate it to the Lupus I cloud, which forms part of the filaments of the shell rim, we fitted an expanding homogeneous spherical shell to the HI data. In addition we used $\mathrm{Na}$ I absorption data in the foreground of stars with known distances from HIPPARCos measurements, using the same shell model as for the HI data. This follows an analysis approach presented elsewhere (Pöppel et al. 2010; Welsh et al. 2010). The parameters of our expanding-shell model are

$$
\text { - distance to the center of the shell } D_{0} \text {; }
$$

1 http://www . iram. fr/IRAMFR/GILDAS

2 http://www . apex-telescope.org/telescope/efficiency 
Table 1. Details of the molecular line maps obtained for Lupus I with APEX-1.

\begin{tabular}{ccccccc}
\hline \hline Cut & Map size $X$ & Map size $Y$ & Length & Center RA $_{\mathrm{J} 2000}$ & Center Dec $_{\mathrm{J} 2000}$ & No. of spectra \\
\hline A & $21^{\prime}$ & $18^{\prime}$ & $26^{\prime}$ & $15: 40: 05.35$ & $-33: 36: 44.4$ & 617 \\
B & $26^{\prime}$ & $6^{\prime}$ & $24^{\prime}$ & $15: 42: 49.80$ & $-34: 08: 37.8$ & 618 \\
C & $13^{\prime}$ & $24^{\prime}$ & $26^{\prime}$ & $15: 44: 57.60$ & $-34: 17: 13.3$ & 620 \\
\hline
\end{tabular}

Notes. The first column gives the name of the cut and the second and third column give the $x$ and $y$ size of the whole map. Column four gives the length of the cut, columns five and six the position of the center of the map, and the last column gives the number of pixels that contain a spectrum. These parameters are valid for both the ${ }^{13} \mathrm{CO}$ and $\mathrm{C}^{18} \mathrm{O}$ maps of one cut. The angular resolution of the maps is $30.1^{\prime \prime}$ and the pixel size $14.3^{\prime \prime}$. The three cuts share a common OFF-position at $\mathrm{RA}=15: 44: 23.605$ and $\mathrm{Dec}=-33: 39: 00.68$.

- galactic longitude $l_{0}$ of the shell center;

- galactic latitude $b_{0}$ of the shell center;

- radius of the inner edge of the shell $r_{\text {in }}$;

- thickness of the shell $\Delta r$;

- density of hydrogen atoms $n_{\mathrm{H}}$ in the shell assumed to be spatially constant;

- expansion velocity of the shell $V_{\text {exp }}$;

- bulk velocity of interstellar medium in the Sco-Cen region $V_{0}$.

The distance to the shell center $D_{0}$ was determined from a multiparameter fit to the absorption data, yielding $D_{0}=138 \pm 26 \mathrm{pc}$. Here, the uncertainties were propagated from the uncertainties in the distance measurements of the stars.

For the fit to the HI data, we transformed this model from 3D Cartesian space $(x, y, z)$ into $(l, b, v)$ space, added the HI Milky Way model of Kalberla et al. (2007) as a large-scale background and optimized model parameters fitting to the HI data by a $\chi^{2}$ minimization. The set of best fitting parameters are:

- $l_{0}=347 \pm 0.5 \mathrm{deg}, b_{0}=25 \pm 0.5 \mathrm{deg}, r_{\text {in }}=(12.3 \pm 0.7) \mathrm{deg}$;

- $\Delta r=2.7 \pm 0.3 \mathrm{deg}, n_{\mathrm{H}}=6 \pm 2 \mathrm{~cm}^{-3}, V_{\exp }=(7 \pm 1) \mathrm{km} \mathrm{s}^{-1}$;

- $V_{0}=8 \pm 1 \mathrm{~km} \mathrm{~s}^{-1}$.

Uncertainties are quoted as $1 \sigma$. The total mass of hydrogen in the shell is $11000 \pm 6000 M_{\odot}$. This is similar to the value determined by Pöppel et al. (2010) from lower quality data.

The mass determination has additional significant systematic uncertainties, as the shell is not detected on the far side. On the near side, a spherical shape is plausibly seen, but the galactic foreground adds uncertainties.

\section{Results}

The individual spectra are complex, with a variety of line profiles. The velocity of the emitting gas ranges from $v_{\mathrm{LSR}}=3.2$ to $6.8 \mathrm{~km} \mathrm{~s}^{-1}$. The maps were convolved with a Gaussian of two pixels or approximately one beam. All observed spectra can be found in Appendix A in Figs. A.2-A.7. The main ${ }^{13} \mathrm{CO}$ emission in cut $\mathrm{A}$ is at velocities of $v_{\mathrm{LSR}}=5.5-6.2 \mathrm{~km} \mathrm{~s}^{-1}$ with the highest intensities between 5.9 and $6.1 \mathrm{~km} \mathrm{~s}^{-1}$. The emission is concentrated at the center of the cut and extends to about $10^{\prime}$ around the center in the channels with highest intensities. Detectable $\mathrm{C}^{18} \mathrm{O}$ emission region is limited to about $2^{\prime}$ around the very center of the cut.

The highest intensities of the three cuts are found in cut B. The bulk of the $\mathrm{C}^{18} \mathrm{O}$ emission lies at the same velocity range as the ${ }^{13} \mathrm{CO}$ emission. It is concentrated on the center of the cut and no emission is found at either edge.

Within cut $\mathrm{C}$ several spatially distinct emission regions can be seen in ${ }^{13} \mathrm{CO}$ and $\mathrm{C}^{18} \mathrm{O}$. In contrast to the other two cuts, the

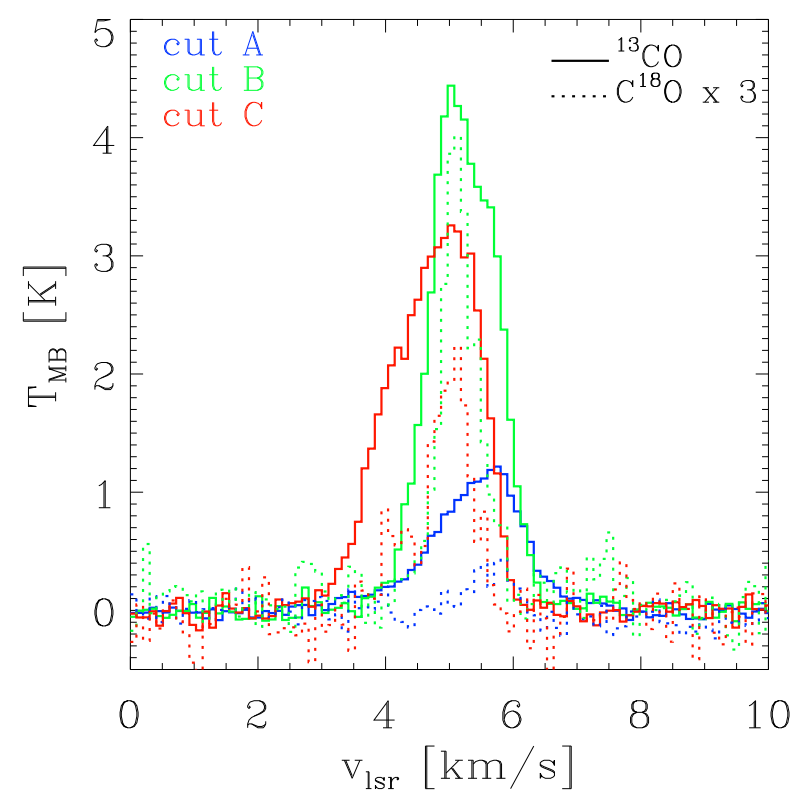

Fig. 2. Average ${ }^{13} \mathrm{CO}$ and $\mathrm{C}^{18} \mathrm{O}$ spectra of the three cuts. The integrated intensities (for $v_{\mathrm{LSR}}=0-10 \mathrm{~km} \mathrm{~s}^{-1}$ ) of the ${ }^{13} \mathrm{CO}$ lines are 1.96, 5.69, and $5.55 \mathrm{~K} \mathrm{~km} \mathrm{~s}^{-1}$ for cut $\mathrm{A}, \mathrm{B}$, and $\mathrm{C}$, respectively. Those of the $\mathrm{C}^{18} \mathrm{O}$ lines are 1.22 , and $0.50 \mathrm{~K} \mathrm{~km} \mathrm{~s}^{-1}$ for cut $\mathrm{B}$ and cut $\mathrm{C}$, respectively.

${ }^{13} \mathrm{CO}$ and $\mathrm{C}^{18} \mathrm{O}$ emission peaks do not overlap, but are located at opposite ends of the cut. $\mathrm{C}^{18} \mathrm{O}$ emission is found at three positions within the cut.

The average ${ }^{13} \mathrm{CO}$ and $\mathrm{C}^{18} \mathrm{O}$ spectrum was then computed for each cut and these spectra are shown in Fig. 2. The solid lines represent the average ${ }^{13} \mathrm{CO}$ spectrum and the dotted lines the average $\mathrm{C}^{18} \mathrm{O}$ spectrum multiplied by three. Cut $\mathrm{A}$ shows the lowest intensities of all three cuts in both lines. The averaged ${ }^{13} \mathrm{CO}$ line is very broad and asymmetric toward velocities lower than the velocity of the peak, i.e., $v_{\mathrm{LSR}}=5.75 \mathrm{~km} \mathrm{~s}^{-1}$. The peak intensity of the ${ }^{13} \mathrm{CO}$ line is $1.22 \mathrm{~K}$. The ${ }^{13} \mathrm{CO}$ line of cut $\mathrm{B}$ is the most narrow and has the highest peak intensity of all three cuts with a value of $4.44 \mathrm{~K}$ at $v_{\mathrm{LSR}}=5.02 \mathrm{~km} \mathrm{~s}^{-1}$. For cut $\mathrm{C}$ the ${ }^{13} \mathrm{CO}$ line also peaks at $v_{\mathrm{LSR}}=5.02 \mathrm{~km} \mathrm{~s}^{-1}$ with a peak intensity of $3.26 \mathrm{~K}$. The averaged $\mathrm{C}^{18} \mathrm{O}$ lines of cut $\mathrm{B}$ and cut $\mathrm{C}$ both peak at $v_{\mathrm{LSR}}=5.13 \mathrm{~km} \mathrm{~s}^{-1}$ and have a peak intensity of 1.34 and $0.74 \mathrm{~K}$, respectively.

\section{Analysis}

We first calculated the optical depth, excitation temperature, and column density under the assumption of local thermodynamical equilibrium (LTE). A multi-Gaussian fit determines the different 
velocity components of the observed spectra. We finally search for a nonthermal contribution to the spectra.

\subsection{Local thermodynamical equilibrium density analysis}

Spectra with a $S / N>4$ were selected to compute optical depth, excitation temperature, and column density. This assures that both lines are clearly detected. For our analysis we assume local thermodynamical equilibrium (LTE) conditions. The excitation temperature $T_{\mathrm{ex}}$ is equal to the kinetic temperature of the gas and is the same for all transitions and both CO isotopologs. This assumption should hold when the temperature distribution along the line of sight is uniform.

The equations and constants used for the calculations of the excitation temperature and column density in this section can be found in Appendix A.

The $\mathrm{H}_{2}$ column density map calculated from dust emission (see Paper I) showed that almost all of Lupus I lies below $1 \times 10^{23} \mathrm{~cm}^{-2}$. Therefore, $\mathrm{C}^{18} \mathrm{O}$ is optically thin (Carlhoff et al. 2013). The optical depth of the ${ }^{13} \mathrm{CO}$ gas is computed (see, e.g., Myers et al. 1983; Ladd et al. 1998) using the relation

$\tau_{13}=\Gamma \times \tau_{18}$,

with $\tau_{13}=\tau\left({ }^{13} \mathrm{CO}\right), \tau_{18}=\tau\left(\mathrm{C}^{18} \mathrm{O}\right)$ and $\Gamma=\left[{ }^{13} \mathrm{CO}\right] /\left[\mathrm{C}^{18} \mathrm{O}\right]$ is the relative abundance ratio of the given isotopologs. Here we adopt a constant abundance ratio of $\Gamma=7.3$ (Wilson \& Rood 1994, local ISM). Then the optical depth $\tau_{13}$ can be approximated by the intensity ratio of the lines

$\frac{T_{\mathrm{mb}}^{13}(v)}{T_{\mathrm{mb}}^{18}(v)}=\frac{1-\exp \left(-\tau_{13}\right)}{1-\exp \left(-\tau_{13} / \Gamma\right)}$,

where $T_{\mathrm{mb}}^{13}(v)$ and $T_{\mathrm{mb}}^{18}(v)$ are the main-beam temperature of the corresponding line at velocity channel $v$. In principle this relation is valid for every velocity channel. Here, we chose $T_{\mathrm{mb}}^{18}(v)=$ $T_{\text {peak }}^{18}$ the peak brightness temperature of the $\mathrm{C}^{18} \mathrm{O}$ line. Then $T_{\mathrm{mb}}^{13}(v)=T_{\text {peak }}^{13}$ is the corresponding brightness temperature of ${ }^{13} \mathrm{CO}$ at the velocity channel of the $\mathrm{C}^{18} \mathrm{O}$ peak intensity. The resulting optical depth maps for $\mathrm{C}^{18} \mathrm{O}$ are shown in the left panels of Fig. A.1. The optical depth $\tau_{18}$ is in the range 0.3 to 1 for most of the cloud with isolated peaks up to about 1.5.

The excitation temperatures are computed using the optically thin $\mathrm{C}^{18} \mathrm{O}$ line (Eqs. (A.1) and (A.2) in the Appendix). The resulting $T_{\mathrm{ex}}$ maps are shown in the middle panels of Fig. A.1. The mean excitation temperatures for cuts A, B, and C are 16.8, 14.7 , and $14.7 \mathrm{~K}$. Our excitation temperatures calculated in our analysis are comparable to previous findings from various $\mathrm{CO}$ and molecular observations of Lupus I. With Eq. (A.3) we compute the $\mathrm{C}^{18} \mathrm{O}$ column density, and find $\mathrm{H}_{2}$ column density assuming a constant abundance ratio of $\left[\mathrm{C}^{18} \mathrm{O}\right] /\left[\mathrm{H}_{2}\right]=1.7 \times 10^{-7}$ (Haworth et al. 2013) for the three column density maps shown in the right panels of Fig. A.1.

The column density distribution (called $N_{\mathrm{H}[\mathrm{CO}]}$ ) has been compared to the total column density obtained from the dust $\left(N_{\mathrm{H} \text { [FIR dust em.] }}\right.$ from Paper I).

We fit a Gaussian to the distribution of the ratio between the $N_{\mathrm{H}[\mathrm{CO}]}$ and $N_{\mathrm{H}[\mathrm{FIR} \text { dustem.] }}$ first considering all the cuts together and then considering each of the three cuts. The distribution peaks at 0.8 with a standard deviation of 0.3 . In general, the column density from $\mathrm{CO}$ gas emission is found to be lower than the column density from dust emission. Along cut A the ratio between gas and dust column density peaks at 1 with a standard deviation $(\sigma)$ of $0.7, \sigma=0.3$ for cut $\mathrm{B}$, while for cut $\mathrm{C}$ the peak of the ratio is not clearly identified as the other two cuts.

\subsection{Determination of different velocity components and nonthermal motions}

In this section we present a detailed analysis of the various $\mathrm{CO}$ velocity components. Each cut was divided into six boxes as shown in Fig. 3. The derived position-velocity (PV) diagrams are shown in Fig. 4. Figure 5 shows the average ${ }^{13} \mathrm{CO}$ (top row) and $\mathrm{C}^{18} \mathrm{O}$ spectrum (bottom row). Using a multicomponent (up to three) Gaussian fit of the spectra (Figs. A.2 to A.7), we computed the peak position and FWHM for each component of the ${ }^{13} \mathrm{CO}$ (top row) and $\mathrm{C}^{18} \mathrm{O}$ (see Table 2 for more details).

We conclude from this that a velocity decrease from cut A to cut $\mathrm{C}$ is seen. No clear trend is seen in line widths, suggesting a homogeneous level of turbulence.

We then derived the nonthermal velocity dispersion of the gas from the measured line width, subtracting the thermal component. Following the standard assumption that the thermal and non-thermal components are independent (see, e.g., Myers 1983), the nonthermal velocity dispersion $\sigma_{\mathrm{NT}}$ is

$\sigma_{\mathrm{NT}}=\sqrt{\frac{\Delta v^{2}}{8 \ln 2}-\frac{k_{\mathrm{B}} T_{\mathrm{kin}}}{m}}$,

where $\Delta v$ is the measured line width, $k_{\mathrm{B}}$ the Boltzmann constant, $T_{\text {kin }}$ the kinetic gas temperature (here we adopt $10 \mathrm{~K}$ ), and $m$ the mass of the observed molecule $\left(m_{{ }^{13} \mathrm{CO}}=29 \mathrm{u}, m_{\mathrm{C}^{18} \mathrm{O}}=30 \mathrm{u}\right)$. Dividing $\sigma_{\mathrm{NT}}$ by the isothermal sound speed $c_{\mathrm{S}}=0.19 \mathrm{~km} \mathrm{~s}^{-1}$ for a $10 \mathrm{~K}$ ISM gas, gives a direct measure of nonthermality of the component. For the measured line widths of ${ }^{13} \mathrm{CO}$ in the three cuts, this indicator is between 2.0 and 4.5, suggesting that the gas would be supersonic. However, correcting the ${ }^{13} \mathrm{CO}$ line width for opacity broadening due to its higher optical depth $\tau_{13}>1-$ 2 (Phillips et al. 1979), while $\mathrm{C}^{18} \mathrm{O} \tau_{18}<1$; this yields a lower indicator value of $\sigma_{\mathrm{NT}} / c_{\mathrm{s}}$ between 0.9 and 1.8. Hence, most of the $\mathrm{C}^{18} \mathrm{O}$ gas within the three cuts is in the trans-sonic regime $\left(1<\sigma_{\mathrm{NT}} / c_{\mathrm{s}}<2\right)$. Typically, the gas of a quiescent cloud resides in the subsonic regime as found, for example, by Hacar \& Tafalla (2011) for L1517. In Lupus I this signature of turbulence might have been imprinted on the gas by the large-scale interaction with the H I shell of USco and the wind-blown bubble of UCL.

\section{Comparison between $\mathrm{CO}$ and $\mathrm{HI}$}

In this section we present a comparison between $\mathrm{CO}$ and H I data.

Figure 6 shows for $v_{\mathrm{LSR}}=0-8 \mathrm{~km} \mathrm{~s}^{-1}$ an H I column density map of Lupus I, together with $\mathrm{C}^{18} \mathrm{O}(1-0)$ emission (Hara et al. 1999) for the same velocity range as the $\mathrm{CO}$ emission. Although the peak velocities of $\mathrm{HI}$ and $\mathrm{CO}$ agree, the $\mathrm{HI}$ and $\mathrm{CO}$ emission do not spatially agree with each other: the highest H I column density is located at the northwestern end of Lupus I, while in the central and the southern part of Lupus I there is less H I emission, roughly by a factor of 1.5 . Between $b=15^{\circ} 30^{\prime}-17^{\circ}$ and $l=$ $338^{\circ} 30^{\prime}-339^{\circ} 30^{\prime}$ the deficit in $\mathrm{HI}$ emission around the centersouth of Lupus I has the form of a little hole. The H I emission, in general, is enhanced at the Galactic west side of Lupus I and has a deficit on its east side with an average $\left\langle N_{\mathrm{H}}\right\rangle=5.5 \times 10^{20} \mathrm{~cm}^{-2}$. The atomic to molecular ratio is poorly defined, given the large uncertainties in the these values. Benedettini et al. (2015), for example, reported a ratio of $\left\langle N_{\mathrm{H}}\right\rangle /\left\langle N_{\mathrm{H}_{2}}\right\rangle \approx 1.6$ calculated from their Herschel column density maps and the H I data of de Geus (1992).

The particular distribution of $\mathrm{CO}$ and $\mathrm{H}$ I shows that Lupus I marks a transition region between the molecular and atomic 
B. Gaczkowski et al.: Lupus I - APEX CO and GASS H I observations
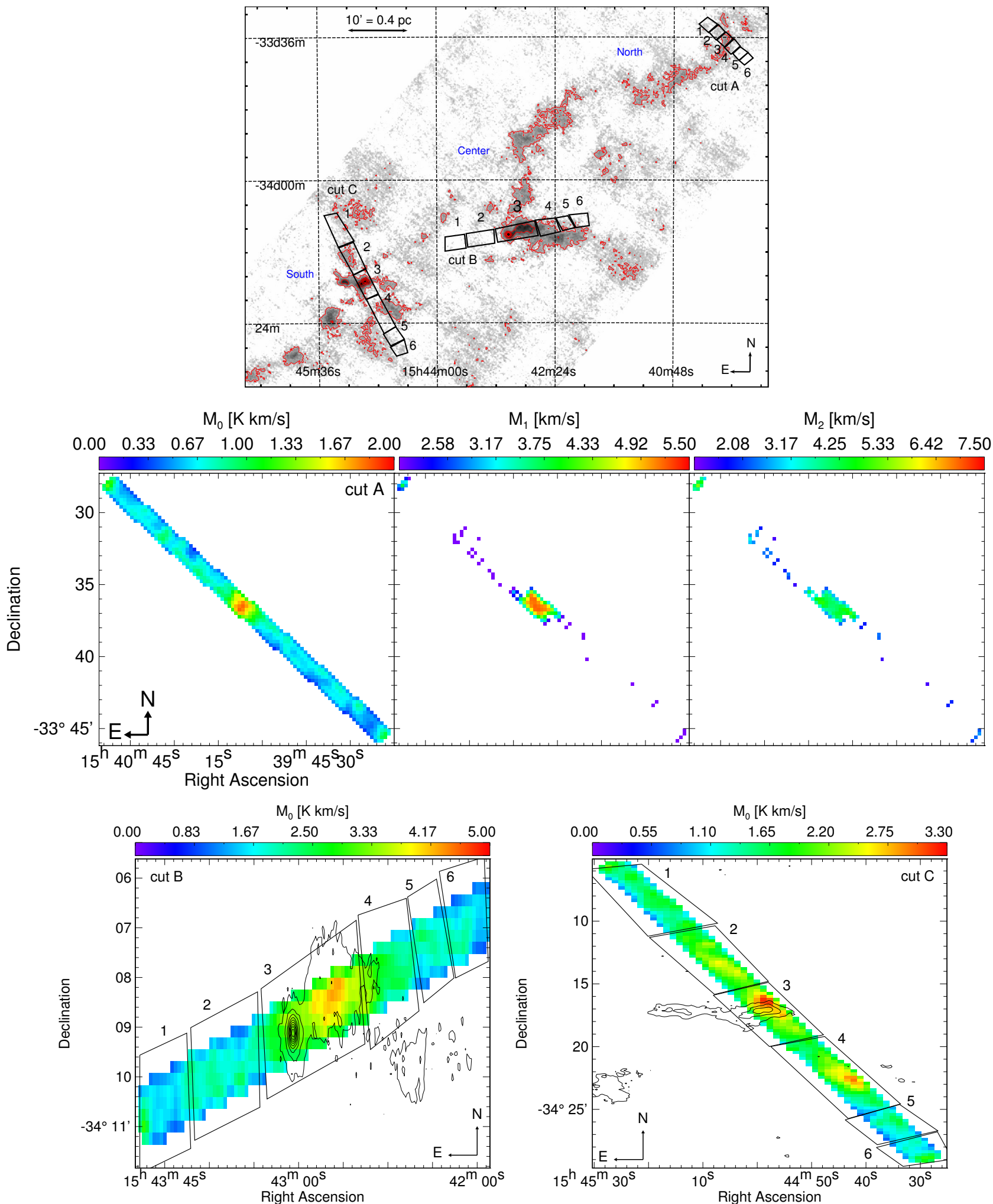

Fig. 3. Top left: dust column density map from LABOCA with red contour levels of $0.3,1,1.7,2.3$, and $3 \times 10^{22} \mathrm{~cm}^{-2}$. Integrated $\mathrm{C}^{18} \mathrm{O}(2-1)$ intensity maps of cut A, B, and C (top right and bottom figures, respectively). The six boxes into which each cut was divided are shown as well as the contours of the LABOCA $870 \mu \mathrm{m}$ continuum emission. Levels are from 10-100\% of the peak intensity of $1.37 \mathrm{Jy} / \mathrm{beam}$.

phase of HI. At those places in the cloud where the atomic hydrogen emission is decreased, the molecular emission is enhanced and vice versa (see Fig. 6). Our analysis shows that the $\mathrm{C}^{18} \mathrm{O}$ emission is significantly higher in the center and south of
Lupus I. We conclude that the north has not built enough dense molecular material yet to start the star formation process. The lower dust column densities seen there coincide with a higher fraction of atomic material. Correspondingly, $\mathrm{C}^{18} \mathrm{O}$ emission is 

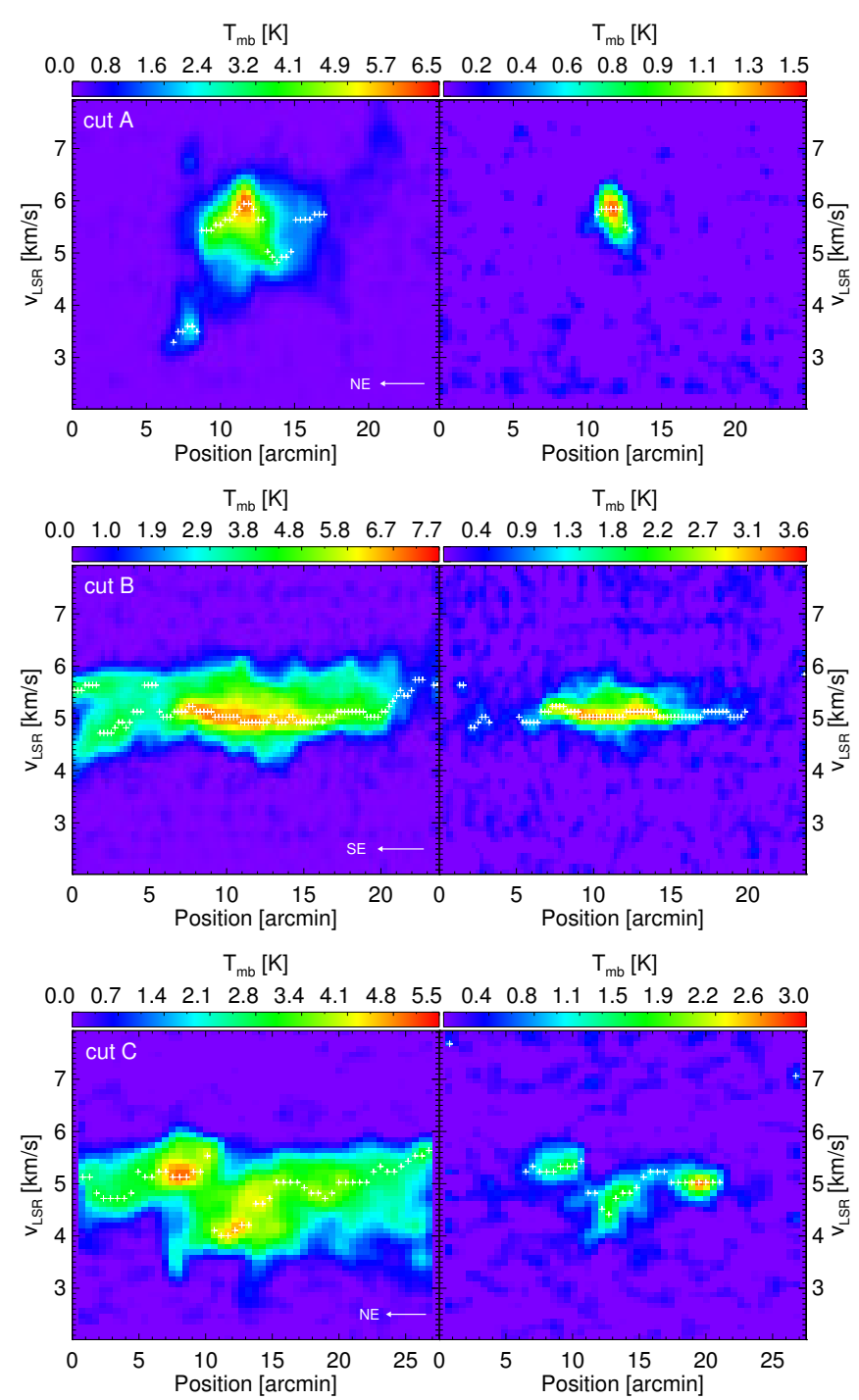

Fig. 4. Position-velocity diagrams of cut A, B, and C (from top to bottom) in ${ }^{13} \mathrm{CO}(2-1)$ (left panel) and $\mathrm{C}^{18} \mathrm{O}(2-1)$ (right panel) along a line through the middle of the cut. The $x$-axis gives the position along this line. The white arrow in the bottom right corner of the left panels indicates the orientation of the cut in the celestial coordinate frame. The white crosses indicate the velocity of the peak intensity at each $x$ position. The cutoff is set to $1 \mathrm{~K}$ in ${ }^{13} \mathrm{CO}(2-1)$ for all three cuts and $0.5 \mathrm{~K}$ in $\mathrm{C}^{18} \mathrm{O}(2-1)$ for cuts $\mathrm{B}$ and $\mathrm{C}$. The $\mathrm{C}^{18} \mathrm{O}(2-1)$ cutoff for cut $\mathrm{A}$ is $0.3 \mathrm{~K}$. All maps have been convolved with a Gaussian of two pixels or approximately one beam.

strongest in the central and southern parts of Lupus I where the $\mathrm{H}$ I emission has a deficit and active star formation and high $\mathrm{CO}$ column densities $\left(N\left(\mathrm{H}_{2}\right) \approx 10^{22} \mathrm{~cm}^{-2}\right)$ can be seen.

Heiner et al. (2015) have created synthetic H I and CO observations from a numerical simulation of decaying turbulence in the thermally bistable neutral medium. They found a power-law tail in the column density PDF when molecular clouds formed. This tail, however, only appears in the PDFs of the molecular material and not the HI. Hence, these authors conclude that the molecule formation is directly correlated with gravitational infall. As the dust PDF of the northern part of Lupus I (see Paper I) does not show a power-law tail, the gravitational infall might not yet be strong enough to convert as much atomic material into molecular in the center-south.

\section{Geometrical model for the interaction zone}

In this section we present a model to check the scenario that Lupus I is located in the interaction zone of the USco H I shell and the UCL wind bubble.

We constructed a geometrical model with the observer located in the origin of the coordinate system that is a 3D Cartesian Galactic coordinate system with

$\left(\begin{array}{l}x \\ y \\ z\end{array}\right)=\left(\begin{array}{c}r \sin \theta \cos \phi \\ r \sin \theta \sin \phi \\ r \cos \theta\end{array}\right)$,

where $\theta=90^{\circ}-b, \phi=l, r$ is the distance, $b$ and $l$ the galactic latitude and longitude, respectively. The $x$-axis points to the Galactic center. Both H I shell and wind bubble are represented by a sphere. We placed the center of the USco sphere at $(l, b)=\left(347^{\circ},+25^{\circ}\right)$ and a distance of $145 \mathrm{pc}$. A radius of $\sim 30 \mathrm{pc}$ is the inner shell radius from our H I model fit (details in Kröll 2018) as observations suggest that Lupus I is located closer to the inner edge of the shell.

We estimated the average projected position of the B stars southwest of Lupus I that might be responsible for the wind bubble (see Paper I) to be $(l, b)=\left(331^{\circ},+12^{\circ}\right)$. This also agrees with the center of a circle in the ROSAT image enclosing the X-ray emission in this region (cf. Fig. 7 in Paper I). The average distance to the stars of the UCL subgroup is about $5 \mathrm{pc}$ smaller than that to USco (de Zeeuw et al. 1999) and thus we placed the center of our UCL sphere model at $140 \mathrm{pc}$.

Our UCL wind bubble is thus on the near side of the USco $\mathrm{H}$ I shell. Figure 7 shows model in three perspectives at the stage at which the UCL bubble touches the USco sphere. In Fig. 7 all points on the surface of the USco sphere that have a model velocity between $3-6 \mathrm{~km} \mathrm{~s}^{-1}$ are marked in cyan. This represents the $\mathrm{CO}$ velocity range we observed in Lupus I. The UCL wind bubble (i.e., the sphere of influence of the massive stars in UCL) interacts with the USco H I shell at positions that have a similar gas velocity to Lupus I.

The Lupus I cloud and the USco H I shell have similar observed velocities and thus it seems reasonable that Lupus I is expanding with the USco shell.

In our purely geometrical model, which does not accurately reflect the physics and resulting complex morphologies present in the Sco-Cen region, the UCL wind bubble and the USco H I shell nevertheless intersect at velocities consistent with our observations of Lupus I. Therefore, the observational data are in agreement with the idea that Lupus I is in an interaction zone between the USco HI shell and the UCL wind bubble. Moreover, this model comparison indicates that Lupus I is expanding with the USco shell and that the cloud is located at the inner edge of the H I shell.

\section{Surroundings of Lupus I and interaction with USco and UCL}

Figure 8 shows the USco H I shell as observed today. A H I column density map of USco and the surroundings of Lupus I are shown in the top panel, together with the dust emission at $850 \mu \mathrm{m}$ and the $\mathrm{CO}(1-0)$ emission from Planck, and early B-type stars in USco and UCL. Our model fit to the HI data yields a shell expansion velocity of $\sim 7 \mathrm{~km} \mathrm{~s}^{-1}$ and a thickness of $\sim 6 \mathrm{pc}$. The current inner and outer radius is $\sim 30 \mathrm{pc}$ and $\sim 36 \mathrm{pc}$, respectively (indicated by the two black circles in Fig. 8). East and west 


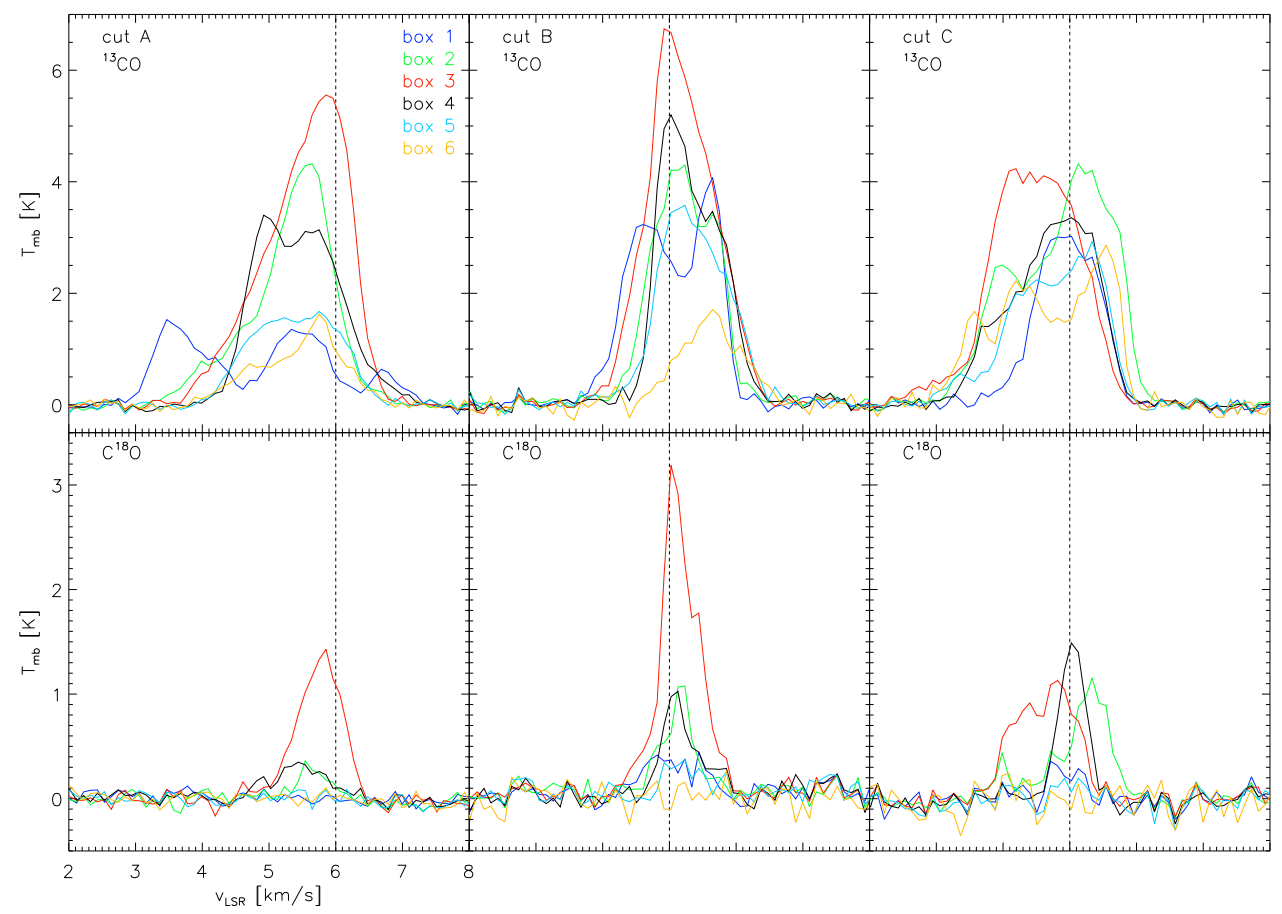

Fig. 5. Averaged spectra within the six boxes into which each cut was divided. The top row shows the average ${ }^{13} \mathrm{CO}(2-1)$ spectra; the bottom shows those of $\mathrm{C}^{18} \mathrm{O}(2-1)$. Each column represents one of the three cuts. The different colors denote the six boxes from 1 to 6 . The vertical dashed line marks for orientation $v_{\mathrm{LSR}}=6 \mathrm{~km} \mathrm{~s}^{-1}$ for cut A and $v_{\mathrm{LSR}}=5 \mathrm{~km} \mathrm{~s}^{-1}$ for cuts B and C.

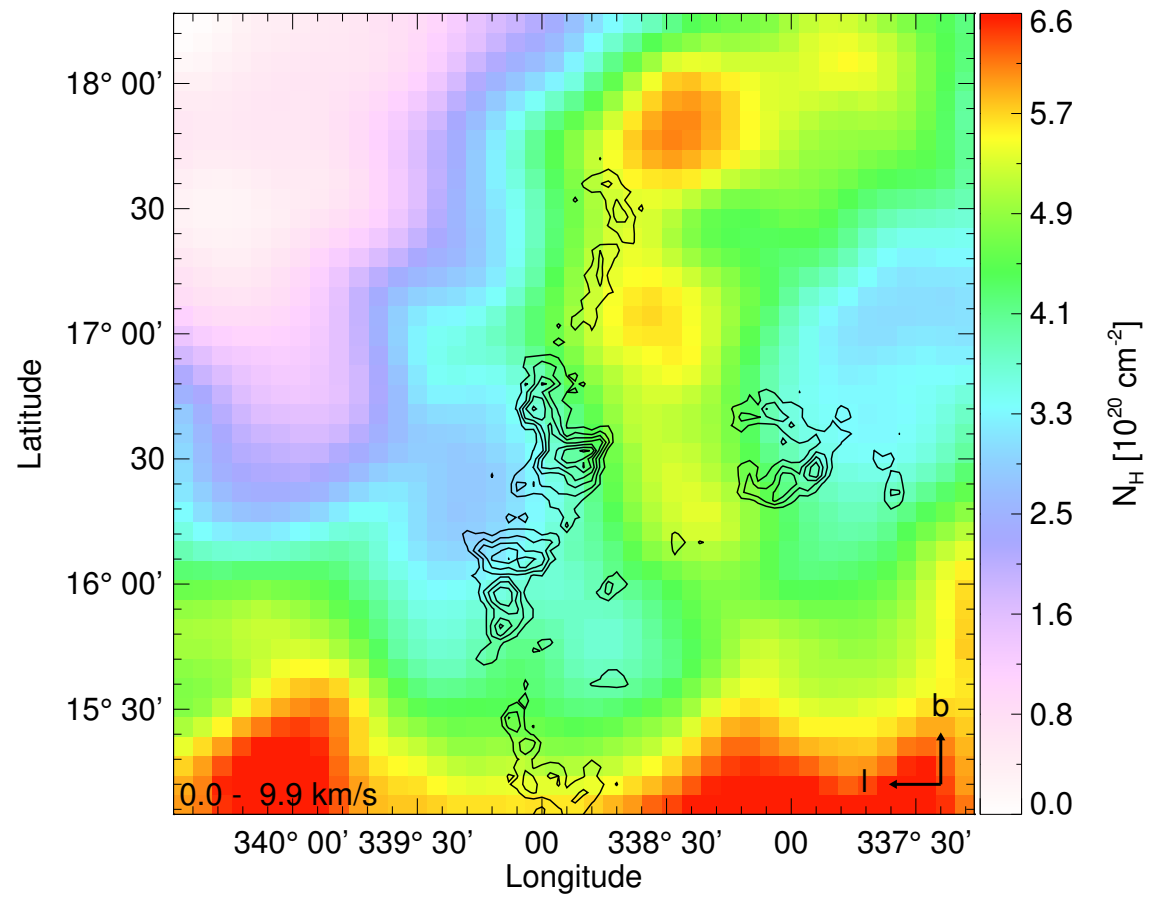

Fig. 6. H I column density map calculated from the integrated emission for $v_{\mathrm{LSR}}=0-10 \mathrm{~km} \mathrm{~s}^{-1}$. Black contours show the integrated $\mathrm{C}^{18} \mathrm{O}(1-0)$ emission observed by Hara et al. (1999).

of Lupus I there are clear H I voids and the atomic material is concentrated in the same area as the dust. A further zoom on Lupus I (right panel of Fig. 8) reveals a complicated distribution of atomic, molecular, and dust material. Whereas the $\mathrm{CO}$ emission is always found in regions of dust emission, there are several areas within the USco shell ridge where there is H I emission but neither dust nor $\mathrm{CO}$ are detected. The layering of atomic and molecular material, as can be seen in Lupus I (molecular gas, traced by $\mathrm{CO}$, is surrounded by $\mathrm{HI}$ ), is also found in some other locations in the shell around Lupus I. In the part of the shell where Lupus I is located, the molecular gas is found preferably behind the atomic gas, i.e., closer to the inner edge of the shell. However, local turbulence and inhomogeneities apparently lead to complex configurations. 

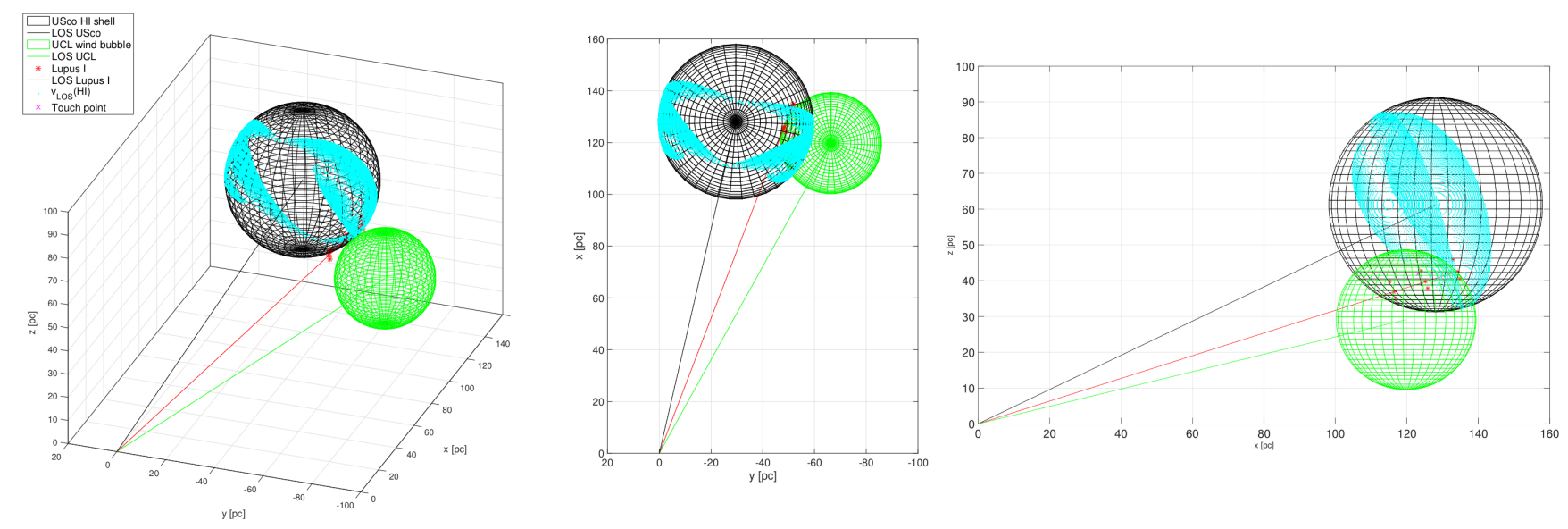

Fig. 7. Geometrical model of the possible interaction zone between the USco H I shell (black sphere) and UCL wind bubble (green sphere). The observer is located in the origin of the coordinate system. The position of Lupus I is marked by three red asterisks that represent the top, middle, and bottom of the main filament, respectively. The cloud is plotted at three different distances of 150,140 , and $130 \mathrm{pc}$ along the red line, which is the line of sight from us to Lupus I. The black and green solid lines are the line of sight from the observer to the corresponding sphere. The cyan dots indicate those positions on the USco shell that have line-of-sight velocities between 3 and $6 \mathrm{~km} \mathrm{~s}^{-1}$, i.e., similar to Lupus I (the white gaps are an effect of the grid model used in the calculations). The magenta cross indicates the touch point between the USco and the UCL sphere. The projections are show in the different panels.

Table 2. Values of peak positions $\left(v_{\mathrm{p}_{i}}^{\mathrm{G}}\right)$ and $\operatorname{FWHM}\left(\Delta v_{i}^{\mathrm{G}}\right)$ of the different components in each averaged spectrum of one box within a cut determined from multicomponent Gaussian fitting (see Figs. A.2 to A.7).

\begin{tabular}{|c|c|c|c|c|c|c|c|c|c|c|}
\hline \multirow{2}{*}{$\begin{array}{c}\text { Box } \\
\#\end{array}$} & \multicolumn{4}{|c|}{$\begin{array}{c}\mathrm{C}^{18} \mathrm{O} \\
{\left[\mathrm{km} \mathrm{s}^{-1}\right]}\end{array}$} & \multicolumn{6}{|c|}{$\begin{array}{c}{ }^{13} \mathrm{CO} \\
{\left[\mathrm{km} \mathrm{s}^{-1}\right]}\end{array}$} \\
\hline & $v_{\mathrm{p}_{1}}^{\mathrm{G}}$ & $\Delta v_{1}^{\mathrm{G}}$ & $v_{\mathrm{p}_{2}}^{\mathrm{G}}$ & $\Delta v_{2}^{\mathrm{G}}$ & $v_{\mathrm{p}_{1}}^{\mathrm{G}}$ & $\Delta v_{1}^{\mathrm{G}}$ & $v_{\mathrm{p}_{2}}^{\mathrm{G}}$ & $\Delta v_{2}^{\mathrm{G}}$ & $v_{\mathrm{p}_{3}}^{\mathrm{G}}$ & $\Delta v_{3}^{\mathrm{G}}$ \\
\hline \multicolumn{11}{|c|}{ cut A } \\
\hline 1 & - & - & - & - & 5.45 & 1.03 & 3.68 & 0.97 & 6.76 & 0.53 \\
\hline 2 & 5.65 & 0.55 & - & - & 5.60 & 0.83 & 4.96 & 1.73 & - & - \\
\hline 3 & 5.77 & 0.76 & - & - & 5.95 & 0.76 & 5.27 & 1.44 & - & - \\
\hline 4 & 5.45 & 0.94 & - & - & 5.63 & 1.22 & 4.86 & 0.56 & - & - \\
\hline 5 & - & - & - & - & 5.86 & 0.88 & 4.96 & 1.00 & - & - \\
\hline 6 & - & - & - & - & 5.76 & 0.87 & 4.75 & 0.87 & - & - \\
\hline$\varnothing$ & 5.62 & 0.75 & - & - & 5.71 & 0.93 & 4.75 & 1.10 & 6.76 & 0.53 \\
\hline \multicolumn{11}{|c|}{ cut B } \\
\hline 1 & 5.03 & 1.08 & - & - & 4.71 & 0.97 & 5.62 & 0.48 & - & - \\
\hline 2 & 5.14 & 0.63 & - & - & 5.13 & 1.00 & 5.75 & 0.30 & - & - \\
\hline 3 & 5.14 & 0.66 & - & - & 5.15 & 1.19 & - & - & - & - \\
\hline 4 & 5.13 & 0.63 & - & - & 5.00 & 0.53 & 5.64 & 0.74 & - & - \\
\hline 5 & 5.25 & 0.81 & - & - & 5.34 & 1.21 & - & - & - & - \\
\hline 6 & - & - & - & - & - & - & 5.62 & 1.10 & - & - \\
\hline$\varnothing$ & 5.14 & 0.76 & - & - & 5.07 & 0.98 & 5.66 & 0.66 & - & - \\
\hline \multicolumn{11}{|c|}{ cut $\mathrm{C}$} \\
\hline 1 & 4.93 & 0.55 & - & - & 4.97 & 1.09 & - & - & - & - \\
\hline 2 & 5.35 & 0.57 & 4.51 & 1.31 & 5.25 & 1.12 & 4.02 & 0.90 & - & - \\
\hline 3 & 4.86 & 0.65 & 4.22 & 0.55 & - & - & 4.50 & 1.51 & - & - \\
\hline 4 & 5.04 & 0.45 & - & - & 5.15 & 0.88 & 4.34 & 1.31 & - & - \\
\hline 5 & - & - & - & - & 5.35 & 0.63 & 4.50 & 1.12 & 3.28 & 0.44 \\
\hline 6 & - & - & - & - & 5.51 & 0.60 & 4.35 & 1.41 & 3.51 & 0.29 \\
\hline$\varnothing$ & 5.05 & 0.56 & 4.37 & 0.93 & 5.25 & 0.86 & 4.34 & 1.25 & 3.40 & 0.37 \\
\hline
\end{tabular}

The comparison of our $\mathrm{CO}$ to $\mathrm{HI}$ data showed that both fall into the same velocity range. If thus Lupus I is co-moving with the atomic gas in the shell its formation within the shell wall seems plausible. Dawson et al. (2011) found that 

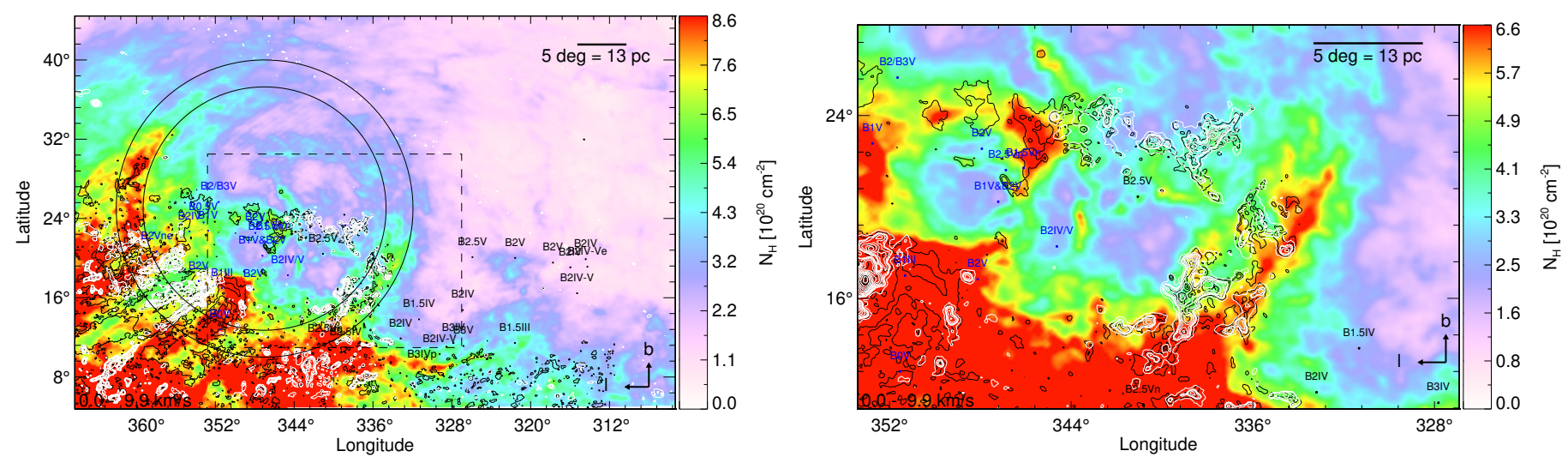

Fig. 8. Left: H I column density map of USco and surroundings of Lupus I calculated from the integrated emission for $v_{\mathrm{LSR}}=0-10 \mathrm{~km} \mathrm{~s}{ }^{-1}$. The two black circles indicate the inner and outer radius of the USco shell from the model fit. The black and white contours represent the dust emission at $850 \mu \mathrm{m}$ and the $\mathrm{CO}(1-0)$ emission from Planck, respectively. The blue and black labeled dots represent early B-type stars in USco and UCL, respectively. Right: zoom into the region indicated as a dashed black box in the left panel.

expanding supershells are capable of sweeping up big amounts of gas from the ISM. Increased density within their walls leads to very short chemical timescales. These authors estimate a transition timescale from atomic to molecular material of $\sim 10^{6}-10^{7} \mathrm{yr}$ (see also Koyama \& Inutsuka 2000; Bergin et al. 2004; Heitsch \& Hartmann 2008; Clark et al. 2012). The estimated age of the USco shell is $~ 5 \mathrm{Myr}$ and hence it should have had sufficient time to accumulate enough atomic gas locally to convert it into molecular gas at the position of Lupus I. From the dust analysis described in Paper I we found that the total cloud (gas + dust) mass of Lupus I is only $\sim 170 M_{\odot}$. This is at least two orders of magnitude lower than the estimated total mass of the USco shell $\left(\sim 10^{4} M_{\odot}\right)$ as derived from the H I model fit. If the atomic gas was homogeneously distributed within the shell, then at least $\sim 10^{3} M_{\odot}$ would have been swept up at the current position of Lupus I, which is enough to create a $\sim 100 M_{\odot}$ molecular cloud, and is similar to the estimate of the total cloud mass by Tachihara et al. (1996). Furthermore, the analysis of the LABOCA continuum data has revealed many pre- and protostellar cores (see Paper I). This suggests that the formation timescale of the cloud and the onset of star formation should be on the order of even less than $2 \mathrm{Myr}$, which is still in agreement with the atomic-to-molecular transition timescale. The large-scale magnetic field is perpendicular to the Lupus I filament, i.e., pointing in the direction of the USco shell expansion. This might have favored the accumulation of cold, dense atomic gas along the field lines and promoted fast molecule formation (Matthews et al. 2014; Hartmann et al. 2001; Vázquez-Semadeni et al. 2011).

The slightly supersonic irregular motions we find are in excellent agreement with predictions for clumps formed by the thin shell instability (Vishniac 1983). Three-dimensional simulations explicitly predict such velocities in clumps formed dynamically in expanding supershells (Krause et al. 2013, Fig. 9). We thus have three pieces of evidence: the correspondence between bulk $\mathrm{HI}$ and molecular velocities; the spatial anticorrelation between molecular and atomic gas; and the magnitude of the irregular motions, which supports the picture that the Lupus I molecular cloud has formed dynamically in the expanding USco supershell. Also X-ray observations suggest that the UCL wind bubble might be colliding with the USco H I shell right at the position of Lupus I, squeezing it in between (see Paper I). This wind bubble, probably still additionally pressurized by past supernova activity, might have provided a counterpressure to the expanding
USco shell and thus favored this position for an additional compression of the shell material. In this way a new molecular cloud could have been created there and this would explain why there are not more very young star forming clouds, except $\rho$ Ophiucus, which is believed to be pre-existing (e.g., Motte et al. 1998), seen distributed within the wall of the USco shell.

This view is also supported by the column density PDFs of the dust emission (see Paper I) with the double-peak profile over the whole extent of the cloud. Together with the dust column density map this shows that Lupus I consists of a dense layer in the center of the filament that is surrounded by much more diffuse material. Simulations have shown that this is consistent with the idea of a two-sided compression through colliding flows (see Matsumoto et al. 2015). Then the molecular cloud is created in a dense and thin sheet that is surrounded by the diffuse material of colliding flows.

This picture does, however, not come without difficulties. A formation of molecular clouds in colliding supershells requires that the shells have the same pressure evolution (Ntormousi et al. 2011). Any pressure difference can lead to cloud ablation and might destroy such clouds within about $10^{6}$ yr (Krause et al. 2013). Since Lupus I is located between USco and UCL, its position also agrees with the much more extended Sco-CMa stream (Bouy \& Alves 2015). It is therefore also possible that pre-existing density enhancements played a role in the formation of Lupus I.

Thompson et al. (2012) detected an increase in star-forming sources at the rim of the shells of HII regions (or IR/Spitzer bubbles). Shells are commonly observed in the Milky Way and other galaxies, but most diffuse shells seen in HI or the infrared do not have obvious triggered star formation. Dense molecular shells and pillars around HII regions often have such triggering (e.g., Elmegreen 2011). Several scenarios attempt to explain star formation associated with expanding shells; these include the scenarios of "collect and collapse" (e.g., Elmegreen \& Lada 1977; Whitworth et al. 1994), "radiative driven implosion" (Deharveng et al. 2010), and enhancement of pre-existing density substructures and subsequent "global implosion" (e.g., Palmeirim et al. 2017; Walch et al. 2015). The trans-sonic irregular motion in Lupus I can be explained by the Vishniac thin shell instability. This supports a picture in which clumping and possibly molecular gas formation was going on for a while, but star formation only set in when 
an external compression, possibly due to a supernova, triggered it. This is also consistent with the double-peaked density PDF in Lupus I and the coeval onset of star formation in the nearby $\rho$ Ophiucus cloud, which could have been triggered by the same event. If the star formation was also related to gradual transformation of gas collected in a shell, then it would be more difficult to understand the sudden and coeval onset of star formation in both Lupus I and $\rho$ Oph. Thus, the data support a more complex, multistage formation process for Lupus I than a simple collect and collapse scenario.

\section{Summary and conclusions}

In this paper we presented new ${ }^{13} \mathrm{CO}(2-1)$ and $\mathrm{C}^{18} \mathrm{O}(2-1)$ line observations of Lupus I with the APEX telescope at three distinct cuts at different parts of the cloud.

A comparison of our $\mathrm{CO}$ data to $\mathrm{HI}$ data yielded that the emission of both tracers lies in the same velocity range. Moreover, $\mathrm{C}^{18} \mathrm{O}$ emission peaks located in the center-south of Lupus I coincide with deficits in $\mathrm{HI}$ emission, whereas the position of the $\mathrm{HI}$ emission peak in the north shows a deficit in $\mathrm{C}^{18} \mathrm{O}$ emission. This resembles two different stages in the transition between atomic and molecular material. In the north, a smaller fraction of atomic hydrogen has been converted into molecular hydrogen and subsequently into dense CO. Therefore, this part of the cloud is quiescent. The center-south of the cloud has instead enough molecular material to form stars actively. This could also be a consequence of the lower dust column density in the northern part of Lupus Ibecause a smaller amount of dust particles reduces the formation efficiency of molecular hydrogen and provides less shielding from destructive UV radiation.

A comparison of the large-scale $\mathrm{HI}, \mathrm{CO}$ and dust emission in the USco shell and in the vicinity of Lupus I revealed that the molecular gas is always found in regions of dust emission and both of those components are preferentially found behind the outer edge of the atomic shell, i.e., further inward. This is the expected configuration for molecular gas being produced inside the expanding $\mathrm{HI}$ shell. The $\mathrm{CO}$ velocities of Lupus I are in the same range as the H I velocities of the USco shell and thus the cloud is co-moving with the shell, which would agree with the formation from the shell material. The timescale of the transition between atomic and molecular gas in such a massive shell with $M \approx 10^{4} M_{\odot}$ (estimated from our model fit to the H I data) was estimated to be $\sim 10^{6}-10^{7} \mathrm{yr}$. This is consistent with the age of the USco shell $(\sim 5 \mathrm{Myr})$ and the age of the young stellar objects in Lupus I ( $<2 \mathrm{Myr}$ ). The UCL wind bubble on the western side of Lupus I is colliding with the USco H I shell at the position of Lupus I, thereby squeezing the region in between. Thus, it acted as a counterpressure to the expanding USco shell and favored this position for an additional compression of the shell material. In this way a new molecular cloud was created there. This additional collisional pressure might explain why there are not more very young star-forming clouds seen distributed within the wall of the USco shell. We show with a geometrical model that the observational data are in agreement with this idea. This model also indicates that Lupus I can be expanding with the USco shell and that the cloud is located at the edge of the inner shell.

We suggest that Lupus I was and is strongly affected by large-scale external compression originating from the expansion of the USco HI shell and the UCL wind bubble and that the cloud was formed out of the atomic material swept up by the USco shell and is now expanding with the shell. Pre-existing gas structure and collision with the UCL wind bubble were likely decisive factors that enhanced a local inhomogeneity and density in the USco shell at a position that favored the formation of Lupus I.

Acknowledgements. We would like to thank the referee for his/her constructive comments, which helped to improve this paper. This work was supported by funding from Deutsche Forschungsgemeinschaft under DFG project numbers PR 569/10-1 and PR 569/10-2 in the context of the Priority Program 1573 "Physics of the Interstellar Medium". Additional support came from funds from the Munich Cluster of Excellence "Origin and Structure of the Universe". The Herschel spacecraft was designed, built, tested, and launched under a contract to ESA managed by the Herschel/Planck Project team by an industrial consortium under the overall responsibility of the prime contractor Thales Alenia Space (Cannes), and including Astrium (Friedrichshafen) responsible for the payload module and for system testing at spacecraft level, Thales Alenia Space (Turin) responsible for the service module, and Astrium (Toulouse) responsible for the telescope, with in excess of a hundred subcontractors. Based on observations obtained with Planck (http://www.esa.int/Planck), an ESA science mission with instruments and contributions directly funded by ESA Member States, NASA, and Canada. HIPE is a joint development by the Herschel Science Ground Segment Consortium, consisting of ESA, the NASA Herschel Science Center, and the HIFI, PACS, and SPIRE consortia. This research has made use of the SIMBAD database, operated at CDS, Strasbourg, France. We acknowledge the use of NASA's SkyView facility (http://skyview.gsfc.nasa.gov) located at NASA Goddard Space Flight Center. M.K. acknowledges support by the Australian Research Council through an Early Career Fellowship, DE130101399.

\section{References}

Benedettini, M., Pezzuto, S., Burton, M. G., et al. 2012, MNRAS, 419, 238 Benedettini, M., Schisano, E., Pezzuto, S., et al. 2015, MNRAS, 453, 2036 Bergin, E. A., Hartmann, L. W., Raymond, J. C., \& Ballesteros-Paredes, J. 2004, ApJ, 612, 921

Blaauw, A. 1964, in The Galaxy and the Magellanic Clouds, ed. F. J. Kerr, IAU Symp., 20, 50

Bouy, H., \& Alves, J. 2015, A\&A, 584, A26

Calderón, D., Ballone, A., Cuadra, J., et al. 2016, MNRAS, 455, 4388

Carlhoff, P., Nguyen Luong, Q., Schilke, P., et al. 2013, A\&A, 560, A24

Clark, P. C., Glover, S. C. O., Klessen, R. S., \& Bonnell, I. A. 2012, MNRAS, 424, 2599

Comerón, F. 2008, Handbook of Star Forming Regions, Vol. II, ed. B. Reipurth, (ASP Monograph Publications), 295

Dawson, J. R., McClure-Griffiths, N. M., Kawamura, A., et al. 2011, ApJ, 728, 127

de Geus, E. J. 1992, A\&A, 262, 258

de Zeeuw, P. T., Hoogerwerf, R., de Bruijne, J. H. J., Brown, A. G. A., \& Blaauw, A. 1999, AJ, 117, 354

Deharveng, L., Schuller, F., Anderson, L. D., et al. 2010, A\&A, 523, A6

Dobbs, C. L., Krumholz, M. R., Ballesteros-Paredes, J., et al. 2014, Protostars and Planets VI (Tucson: University of Arizona Press), 3

Elmegreen, B. G. 2011, in EAS Pub. Ser. 51, eds. C. Charbonnel, \& T. Montmerle, 45

Elmegreen, B. G., \& Lada, C. J. 1977, ApJ, 214, 725

Gaczkowski, B., Preibisch, T., Stanke, T., et al. 2015, A\&A, 584, A36

Goldsmith, P. F., \& Langer, W. D. 1999, ApJ, 517, 209

Güsten, R., Nyman, L. A., Schilke, P., et al. 2006, A\&A, 454, L13

Hacar, A., \& Tafalla, M. 2011, A\&A, 533, A34

Hara, A., Tachihara, K., Mizuno, A., et al. 1999, PASJ, 51, 895

Hartmann, L., Ballesteros-Paredes, J., \& Bergin, E. A. 2001, ApJ, 562, 852

Haworth, T. J., Harries, T. J., Acreman, D. M., \& Rundle, D. A. 2013, MNRAS, 431, 3470

Heiner, J. S., Vázquez-Semadeni, E., \& Ballesteros-Paredes, J. 2015, MNRAS, 452, 1353

Heitsch, F., \& Hartmann, L. 2008, ApJ, 689, 290

Kalberla, P. M. W., Dedes, L., Kerp, J., \& Haud, U. 2007, A\&A, 469, 511

Kalberla, P. M. W., McClure-Griffiths, N. M., Pisano, D. J., et al. 2010, A\&A, 521, A17

Koyama, H., \& Inutsuka, S.-I. 2000, ApJ, 532, 980

Krause, M., Fierlinger, K., Diehl, R., et al. 2013, A\&A, 550, A49

Ladd, E. F., Fuller, G. A., \& Deane, J. R. 1998, ApJ, 495, 871

Mangum, J. G., \& Shirley, Y. L. 2015, PASP, 127, 266

Matsumoto, T., Dobashi, K., \& Shimoikura, T. 2015, ApJ, 801, 77

Matthews, T. G., Ade, P. A. R., Angilè, F. E., et al. 2014, ApJ, 784, 116

Motte, F., Andre, P., \& Neri, R. 1998, A\&A, 336, 150

Myers, P. C. 1983, ApJ, 270, 105

Myers, P. C., Linke, R. A., \& Benson, P. J. 1983, ApJ, 264, 517

Ntormousi, E., Burkert, A., Fierlinger, K., \& Heitsch, F. 2011, ApJ, 731, 13

Palmeirim, P., Zavagno, A., Elia, D., et al. 2017, A\&A, 605, A35 
B. Gaczkowski et al.: Lupus I - APEX CO and GASS H I observations

Phillips, T. G., Huggins, P. J., Wannier, P. G., \& Scoville, N. Z. 1979, ApJ, 231, 720

Pöppel, W. G. L., Bajaja, E., Arnal, E. M., \& Morras, R. 2010, A\&A, 512, A83 Preibisch, T., \& Mamajek, E. 2008, Handbook of Star Forming Regions, Vol. II, ed. B. Reipurth, (ASP Monograph Publications), 235

Preibisch, T., Brown, A. G. A., Bridges, T., Guenther, E., \& Zinnecker, H. 2002, AJ, 124, 404

Tachihara, K., Dobashi, K., Mizuno, A., Ogawa, H., \& Fukui, Y. 1996, PASJ, 48, 489

Tachihara, K., Toyoda, S., Onishi, T., et al. 2001, PASJ, 53, 1081

Thompson, M. A., Urquhart, J. S., Moore, T. J. T., \& Morgan, L. K. 2012, MNRAS, 421, 408

Tothill, N. F. H., Löhr, A., Parshley, S. C., et al. 2009, ApJS, 185, 98
Vassilev, V., Meledin, D., Lapkin, I., et al. 2008, A\&A, 490, 1157

Vázquez-Semadeni, E., Banerjee, R., Gómez, G. C., et al. 2011, MNRAS, 414, 2511

Vilas-Boas, J. W. S., Myers, P. C., \& Fuller, G. A. 2000, ApJ, 532, 1038

Vishniac, E. T. 1983, ApJ, 274, 152

Walch, S., Whitworth, A. P., Bisbas, T. G., Hubber, D. A., \& Wünsch, R. 2015, MNRAS, 452, 2794

Welsh, B. Y., Lallement, R., Vergely, J.-L., \& Raimond, S. 2010, A\&A, 510, A54 Whitworth, A. P., Bhattal, A. S., Chapman, S. J., Disney, M. J., \& Turner, J. A. 1994, MNRAS, 268, 291

Wilson, T. L., \& Rood, R. 1994, ARA\&A, 32, 191

Wilson, T. L., Rohlfs, K., \& Huttemeister, S. 2012, Tools of Radio Astronomy, 5th edn. (Moscow: Fizmatlit) 


\section{Appendix A: Calculations of gas temperatures and column densities}

\section{A.1. Excitation temperature}

The excitation temperature can be calculated via

$$
T_{\mathrm{ex}}(\tau)=\frac{T_{v}^{18}}{\ln \left\{1+\left[\frac{T_{\text {peak }}^{18}}{T_{v}^{18}}\left(1-\exp \left(-\tau_{18}\right)\right)^{-1}+J\left(T_{\mathrm{bg}}\right) / T_{v}^{18}\right]^{-1}\right\}},
$$

where

$J(T) \equiv \frac{c^{2}}{2 k_{\mathrm{B}} v^{2}} B_{v}(T)=T_{v}\left[\exp \left(\mathrm{T}_{v} / \mathrm{T}\right)-1\right]^{-1}$,

$T_{v}=\frac{h v_{0}}{k_{\mathrm{B}}}$, and $T_{\mathrm{bg}}=2.73 \mathrm{~K}$ is the temperature of the cosmic microwave background (CMB). Equations (A.1), (A.2) are derived from the equations given in Wilson et al. (2012).

Table A.1. Constants of the ${ }^{13} \mathrm{CO}(2-1)$ and $\mathrm{C}^{18} \mathrm{O}(2-1)$ lines that are needed for the calculations in this section.

\begin{tabular}{ccccc}
\hline \hline Line & $\begin{array}{c}v_{0} \\
{[\mathrm{GHz}]}\end{array}$ & $\begin{array}{c}T_{v} \\
{[\mathrm{~K}]}\end{array}$ & $\begin{array}{c}\mu \\
{[\mathrm{D}]}\end{array}$ & $\begin{array}{c}B_{\text {rot }} \\
{\left[10^{10} \mathrm{~Hz}\right]}\end{array}$ \\
\hline${ }^{13} \mathrm{CO}(2-1)$ & 220.3986765 & 10.577469 & 0.122 & 5.509967 \\
$\mathrm{C}^{18} \mathrm{O}(2-1)$ & 219.5603568 & 10.537236 & 0.110 & 5.489009 \\
\hline
\end{tabular}

Notes. $v_{0}$ is the rest frequency of the line, $T_{v}=h v_{0} / k_{\mathrm{B}}, \mu$ is the dipole moment of the molecule, and $B_{\text {rot }}$ its rotational constant.

\section{A.2. $\mathrm{H}_{2}$ column density}

For the calculation of the column density we used

$N\left(\mathrm{C}^{18} \mathrm{O}\right)=\frac{\tau_{18}}{1-\exp \left(-\tau_{18}\right)} f\left(T_{\mathrm{ex}}\right) \int T_{\mathrm{mb}}^{18} \mathrm{~d} v$

from Carlhoff et al. (2013), where the functions $f\left(T_{\mathrm{ex}}\right)$ and $Q\left(T_{\text {ex }}\right)$ are defined as

$f\left(T_{\mathrm{ex}}\right)=\frac{3 h}{8 \pi^{3} \mu^{2}} Q\left(T_{\mathrm{ex}}\right)\left\{\left[\left(J\left(T_{\mathrm{ex}}\right)-J\left(T_{\mathrm{bg}}\right)\right]\left[1-\exp \left(-\frac{T_{v}^{18}}{T_{\mathrm{ex}}}\right)\right]\right\}^{-1}\right.$,

$Q\left(T_{\mathrm{ex}}\right)=\frac{k_{\mathrm{B}} T_{\mathrm{ex}}}{J_{\mathrm{u}} B_{\mathrm{rot}} h} \exp \left(\frac{B_{\mathrm{rot}} J_{\mathrm{u}}\left(J_{\mathrm{u}}+1\right) h}{k_{\mathrm{B}} T_{\mathrm{ex}}}\right)$

with $J_{\mathrm{u}}=2$ the upper level of the $J=2 \rightarrow 1$ transition, $\mu$ the dipole moment of the molecule, and $B_{\text {rot }}$ its rotational constant. The partition function $Q\left(T_{\mathrm{ex}}\right)$ is approximated for a linear molecule (Mangum \& Shirley 2015). The factor $\tau_{18} /\left(1-\exp \left(-\tau_{18}\right)\right)$ corrects the integrated intensity for possible opacity broadening of the line (see Goldsmith \& Langer 1999). But this effect should be in any case small for the $\mathrm{C}^{18} \mathrm{O}$ line because $\tau_{18}$ stays below unity in most pixels. 

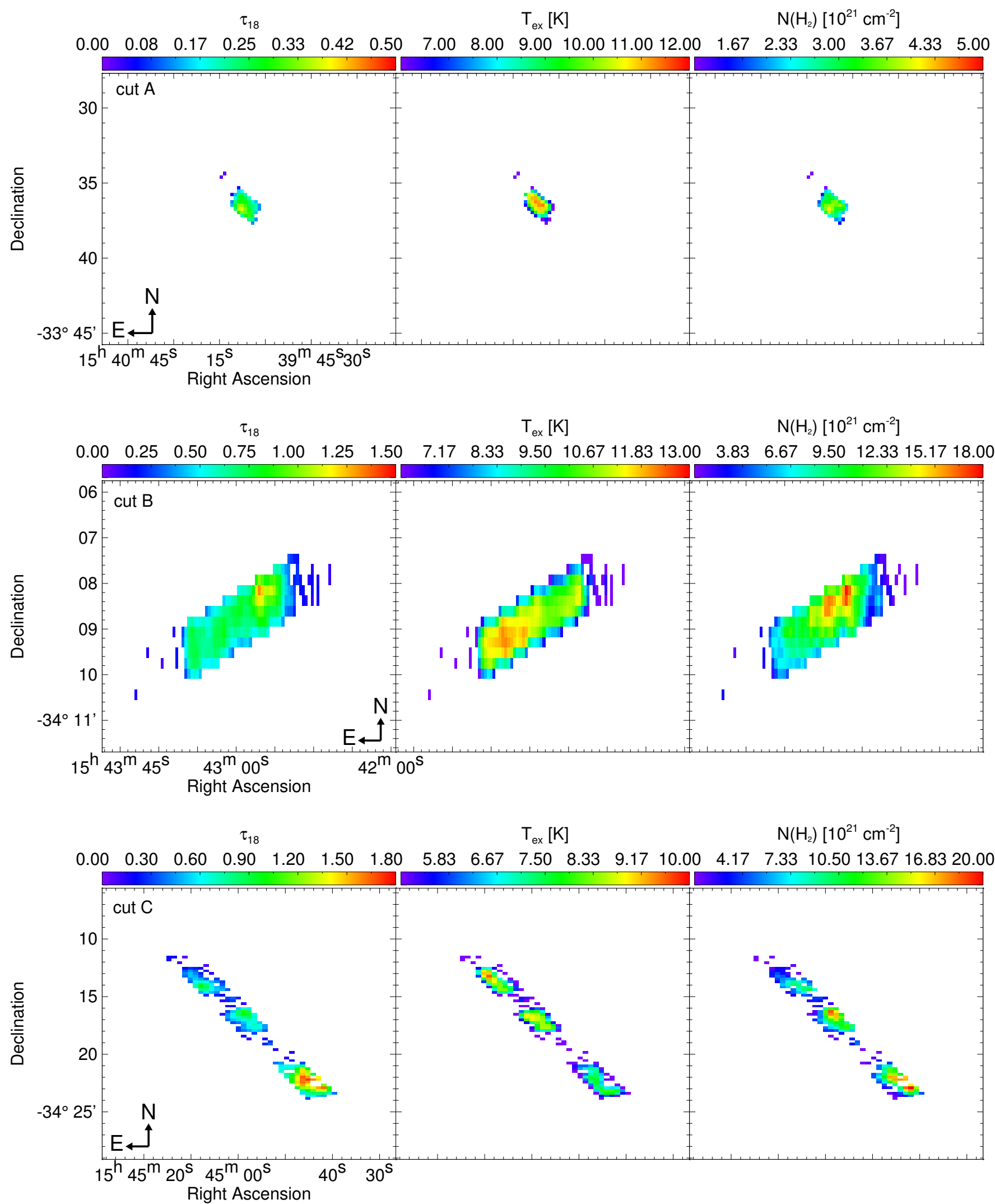

Fig. A.1. Results of the LTE analysis for cut A, B, and C (from top to bottom). The left panel shows the optical depth $\tau_{18}$, the middle panel the excitation temperature $T_{\mathrm{ex}}$, and the right panel the $\mathrm{H}_{2}$ column density. All maps were convolved with a Gaussian of two pixels that corresponds approximatively to the beam size. Pixels within each cut for which the ${ }^{13} \mathrm{CO}(2-1)$ and the $\mathrm{C}^{18} \mathrm{O}(2-1)$ spectrum had a $S / N<4$ are left blank. 

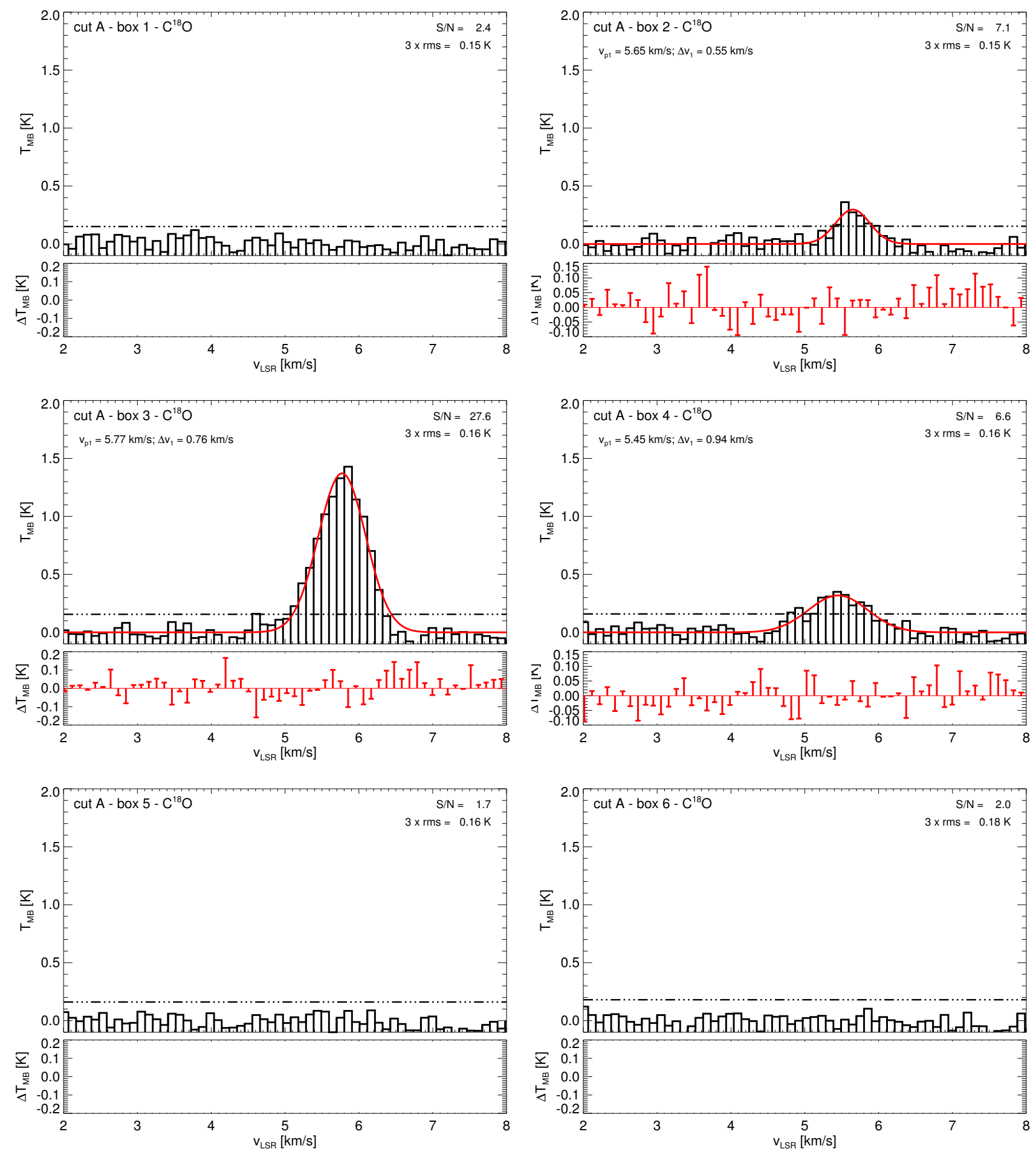

Fig. A.2. Histograms of the average $\mathrm{C}^{18} \mathrm{O}(2-1)$ spectra of the six boxes across cut A with a bin size of $0.1 \mathrm{~km} \mathrm{~s}^{-1}$. The black dash-dotted line indicates the $3 \times \mathrm{rms}$ limit. The values of $3 \times \mathrm{rms}$ and the signal-to-noise ratio $\mathrm{S} / \mathrm{N}$ are given in the upper right of each plot. The red solid line shows the Gaussian fit to the spectrum. If more than one component was fitted, each of the components is represented by a blue dashed line and the red solid line is their sum. The residuals of the fit are given by the red bars in the small plot below each spectrum. Peak positions $v_{\mathrm{p}_{i}}$ and FWHM $\Delta v_{i}$ of each component in one box are given in the upper left of each plot. A summary of all these values can be found in Table 2, where they are denoted by an upper index $\mathrm{G}$. 

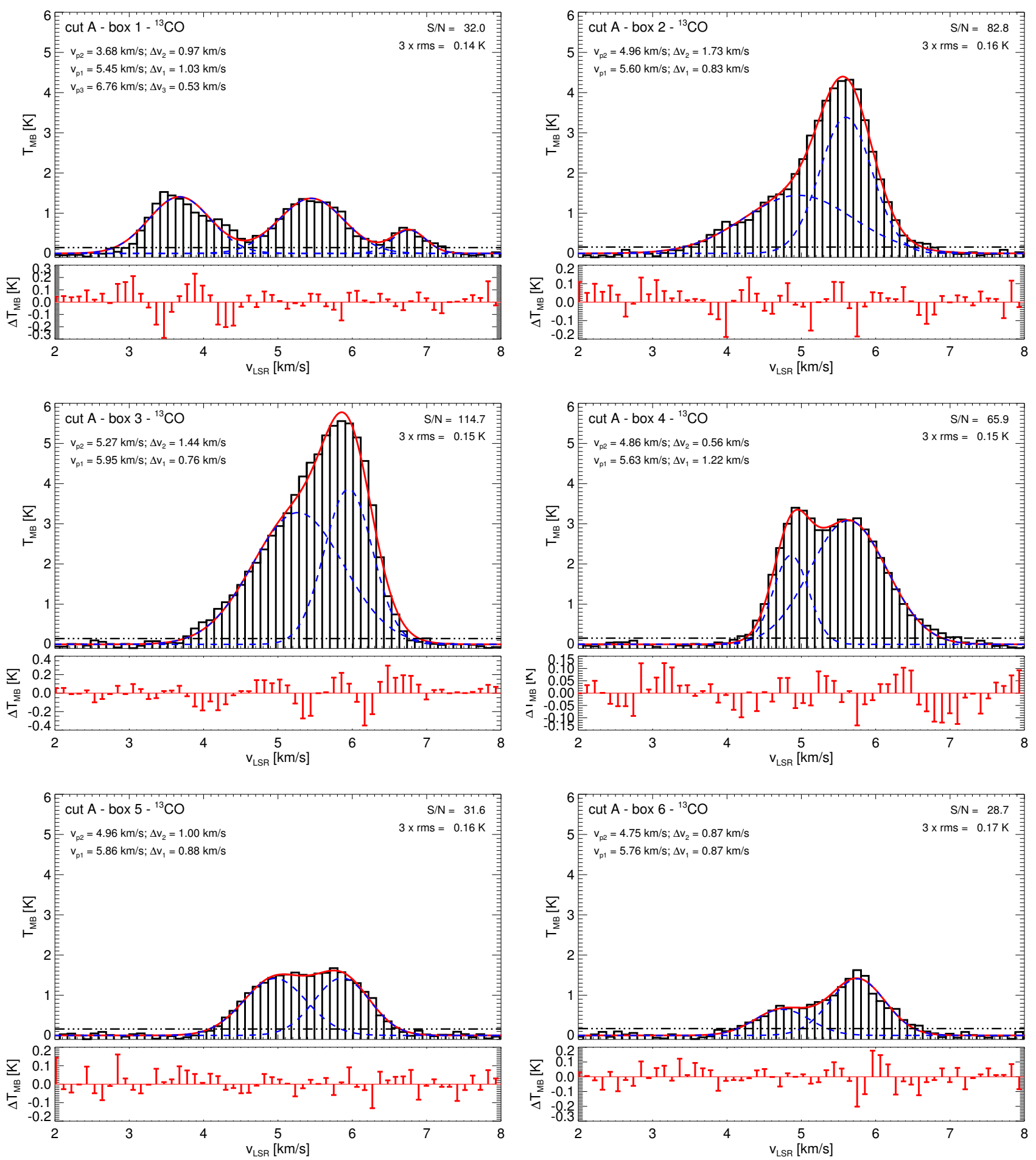

Fig. A.3. Histograms of the average ${ }^{13} \mathrm{CO}(2-1)$ spectra of the six boxes across cut A with a bin size of $0.1 \mathrm{~km} \mathrm{~s}^{-1}$. The black dash-dotted line indicates the $3 \times \mathrm{rms}$ limit. The values of $3 \times \mathrm{rms}$ and the signal-to-noise ratio $\mathrm{S} / \mathrm{N}$ are given in the upper right of each plot. The red solid line shows the Gaussian fit to the spectrum. If more than one component was fitted, each of the components is represented by a blue dashed line and the red solid line is their sum. The residuals of the fit are given by the red bars in the small plot below each spectrum. Peak positions $v_{\mathrm{p}_{i}}$ and FWHM $\Delta v_{i}$ of each component in one box are given in the upper left of each plot. A summary of all these values can be found in Table 2 , where they are denoted by an upper index $\mathrm{G}$. 

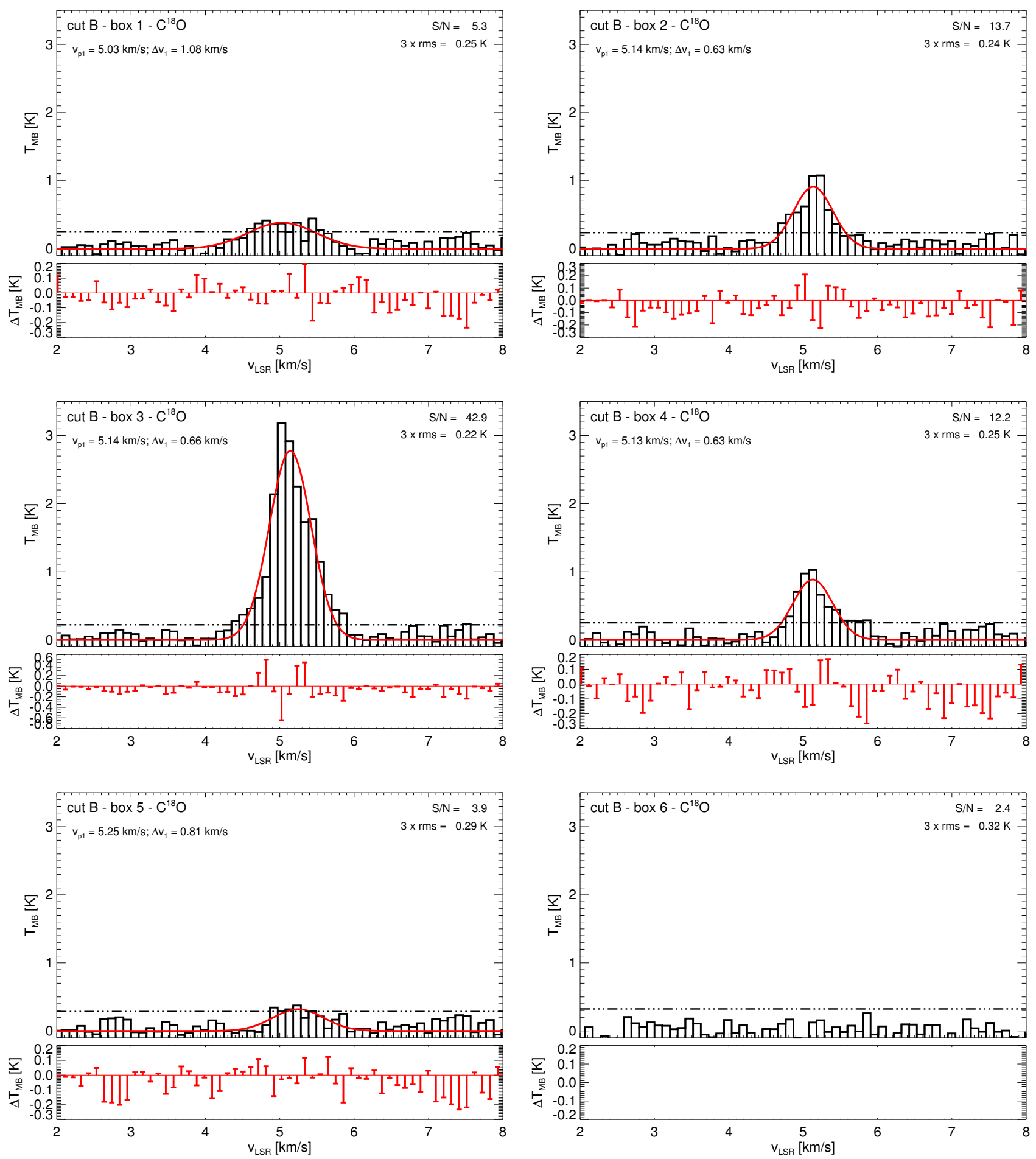

Fig. A.4. Same as Fig. A.2 but for cut B. 

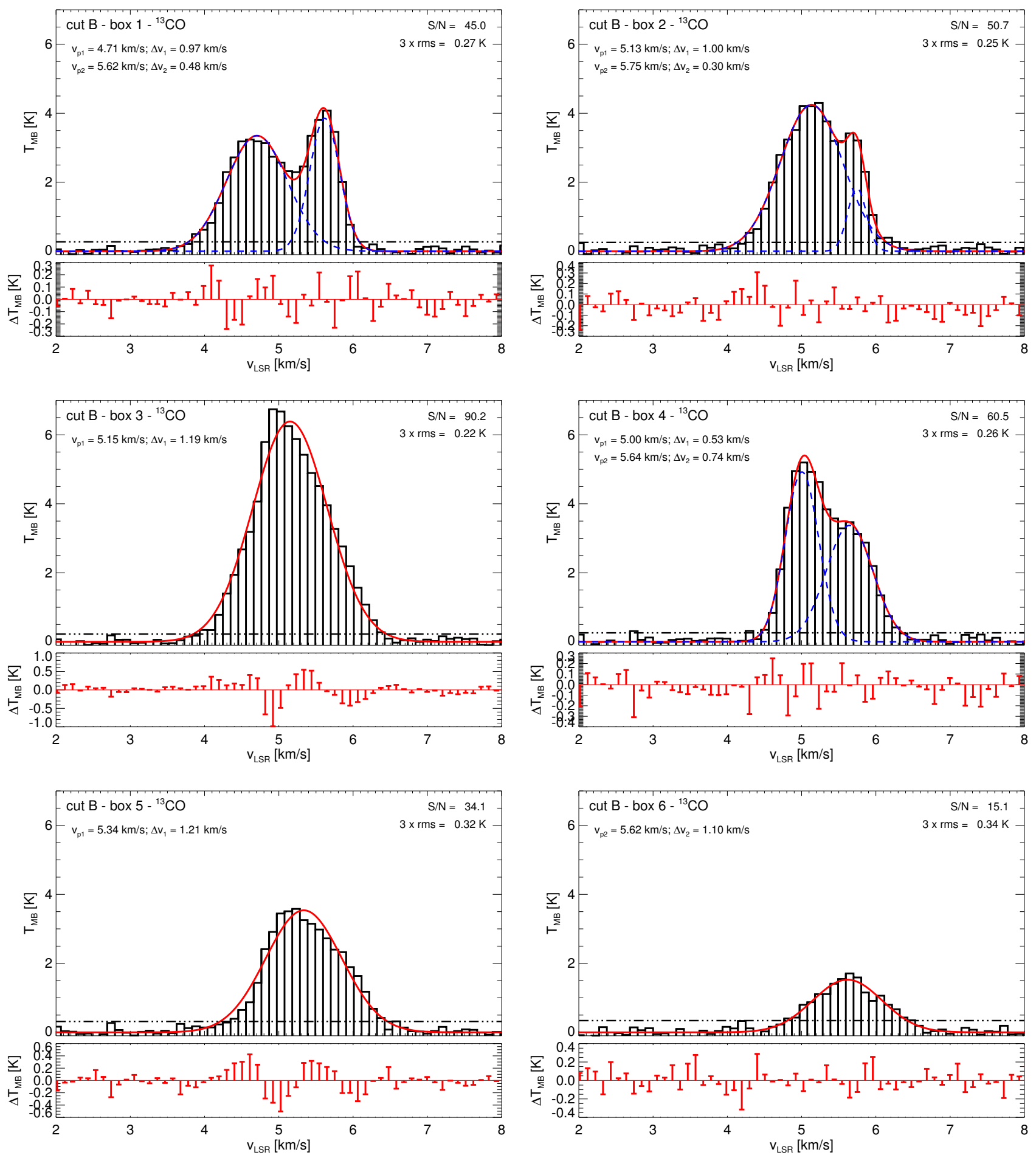

Fig. A.5. Same as Fig. A.3 but for cut B. 

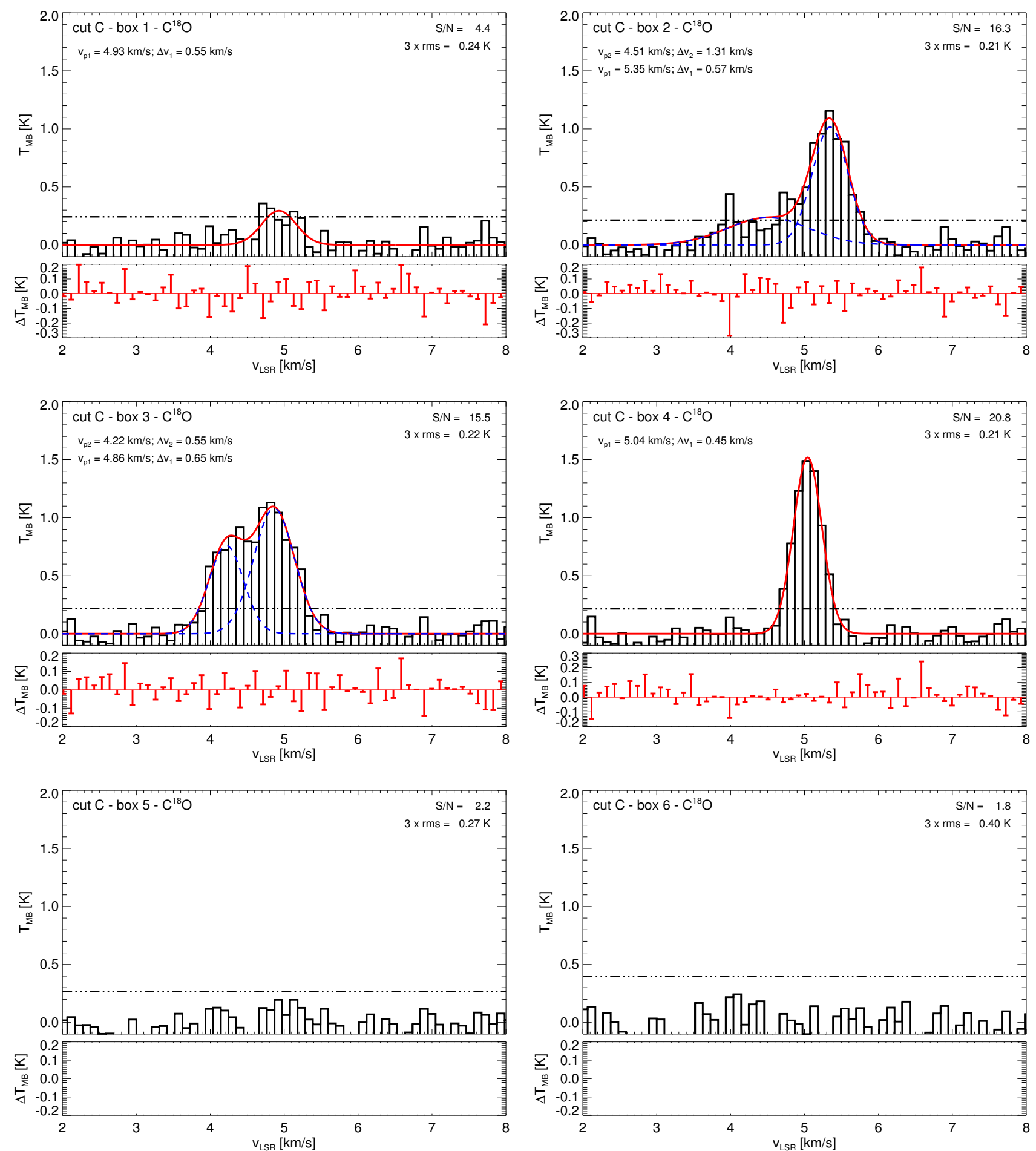

Fig. A.6. Same as Fig. A.2 but for cut C. 

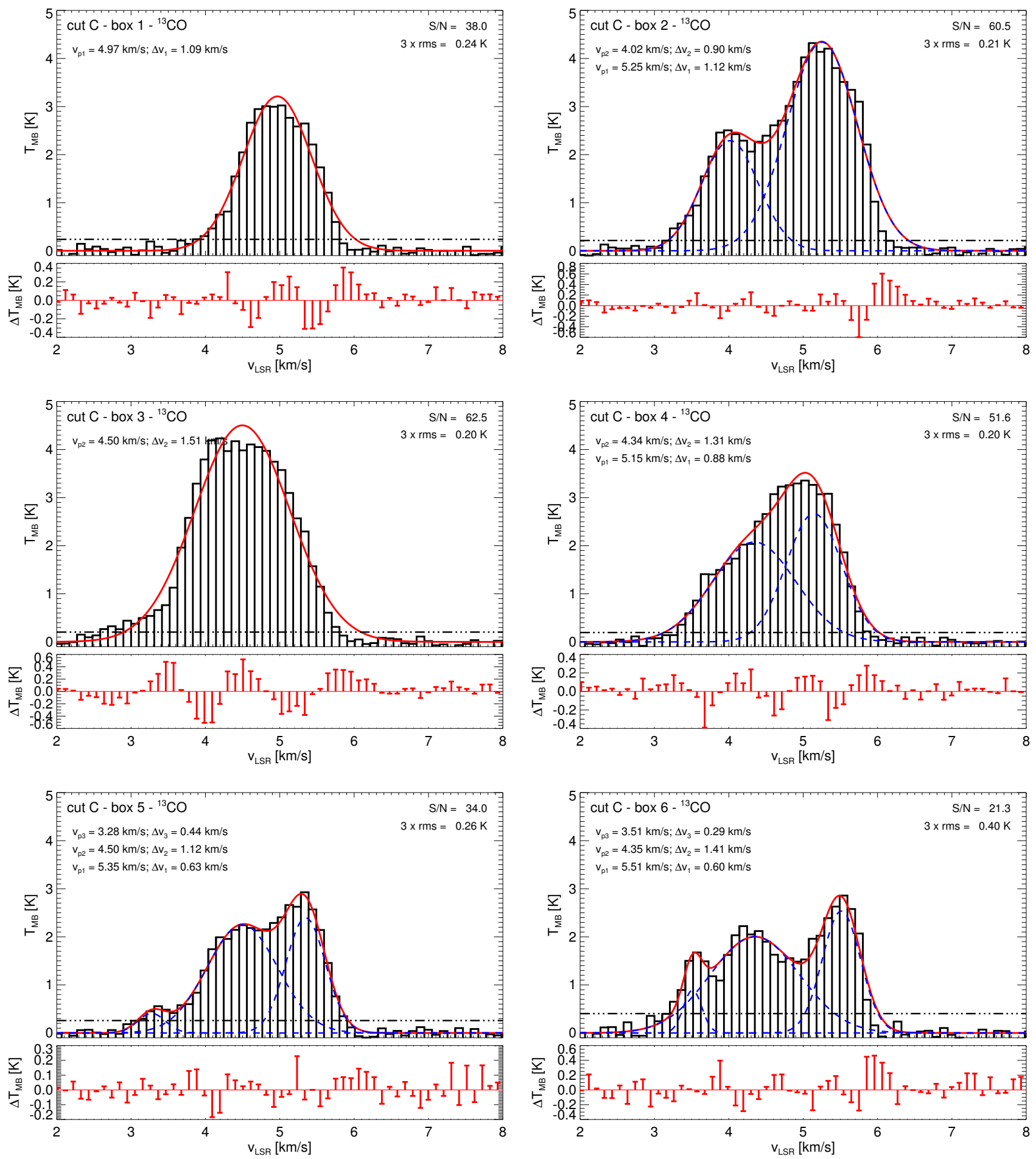

Fig. A.7. Same as Fig. A.3 but for cut C. 